\title{
eHealth in Geriatric Rehabilitation: Systematic Review of Effectiveness, Feasibility, and Usability
}

Jules J M Kraaijkamp ${ }^{1,2}$, MSc; Eléonore F van Dam van Isselt ${ }^{1}$, MD, PhD; Anke Persoon ${ }^{3}$, PhD; Anke Versluis ${ }^{1}$, $\mathrm{PhD}$; Niels H Chavannes ${ }^{1}$, MD, PhD; Wilco P Achterberg ${ }^{1}$, MD, PhD

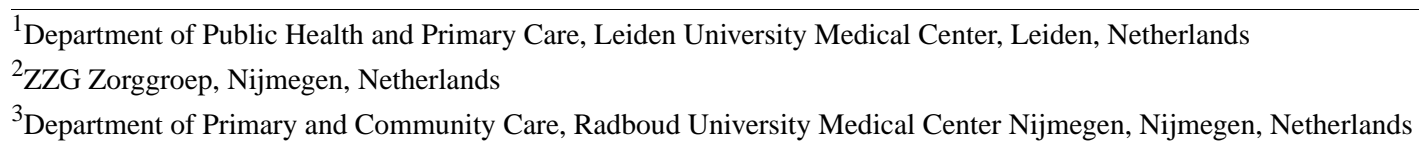

Corresponding Author:

Jules J M Kraaijkamp, MSc

Department of Public Health and Primary Care

Leiden University Medical Center

Hippocratespad 21

Leiden, $2333 \mathrm{ZD}$

Netherlands

Phone: 31610599869

Email: J.J.M.Kraaijkamp@lumc.nl

\section{Abstract}

Background: eHealth has the potential to improve outcomes such as physical activity or balance in older adults receiving geriatric rehabilitation. However, several challenges such as scarce evidence on effectiveness, feasibility, and usability hinder the successful implementation of eHealth in geriatric rehabilitation.

Objective: The aim of this systematic review was to assess evidence on the effectiveness, feasibility, and usability of eHealth interventions in older adults in geriatric rehabilitation.

Methods: We searched 7 databases for randomized controlled trials, nonrandomized studies, quantitative descriptive studies, qualitative research, and mixed methods studies that applied eHealth interventions during geriatric rehabilitation. Included studies investigated a combination of effectiveness, usability, and feasibility of eHealth in older patients who received geriatric rehabilitation, with a mean age of $\geq 70$ years. Quality was assessed using the Mixed Methods Appraisal Tool and a narrative synthesis was conducted using a harvest plot.

Results: In total, 40 studies were selected, with clinical heterogeneity across studies. Of 40 studies, 15 studies (38\%) found eHealth was at least as effective as non-eHealth interventions (56\% of the 27 studies with a control group), 11 studies (41\%) found eHealth interventions were more effective than non-eHealth interventions, and 1 study (4\%) reported beneficial outcomes in favor of the non-eHealth interventions. Of 17 studies, 16 (94\%) concluded that eHealth was feasible. However, high exclusion rates were reported in 7 studies of $40(18 \%)$. Of 40 studies, $4(10 \%)$ included outcomes related to usability and indicated that there were certain aging-related barriers to cognitive ability, physical ability, or perception, which led to difficulties in using eHealth.

Conclusions: eHealth can potentially improve rehabilitation outcomes for older patients receiving geriatric rehabilitation. Simple eHealth interventions were more likely to be feasible for older patients receiving geriatric rehabilitation, especially, in combination with another non-eHealth intervention. However, a lack of evidence on usability might hamper the implementation of eHealth. eHealth applications in geriatric rehabilitation show promise, but more research is required, including research with a focus on usability and participation.

(J Med Internet Res 2021;23(8):e24015) doi: 10.2196/24015

\section{KEYWORDS}

geriatric rehabilitation; eHealth; mHealth; digital health; effectiveness; feasibility; usability; systematic review 


\section{Introduction}

The world's population is aging rapidly. Currently, 143 million people are aged 80 years or older, and this number is expected to rise to around 426 million in 2050 [1]. Although many older adults are relatively fit, functional decline, multimorbidity, and geriatric syndromes such as frailty or falls are common in older adults $[2,3]$. A combination of these age-associated conditions triggers an increased risk of adverse outcomes such as hospitalization, functional impairments, and even mortality [4]. Postacute care such as geriatric rehabilitation aims to diminish these age-associated risks. Evidence shows that geriatric rehabilitation can improve functional outcomes and reduce nursing home admissions and mortality $[5,6]$. On the other hand, the rapidly aging populations and lack of staff are putting pressure on the quality, accessibility, and affordability of geriatric rehabilitation. In regard to these problems, the use of eHealth can be seen as important and promising, as it has the potential to simultaneously improve both rehabilitation outcomes and efficiency.

eHealth can be defined as "the use of digital information and communication to support and/or improve health and health care" [7]. Some examples of eHealth are video communication, exergames (ie, active video games), and mobile apps. Although eHealth can be seen as important and promising, successful implementation of eHealth interventions in geriatric rehabilitation is complex, can be time consuming, and involves a variety of determinants on multiple levels [8-10]. To safely and successfully implement eHealth in geriatric rehabilitation, scientific evaluation of eHealth is key [11,12]. Three important outcome measures for the evaluation of eHealth in geriatric rehabilitation can be identified: effectiveness, feasibility, and usability $[9,13]$.

In terms of effectiveness, previous reviews show that eHealth can improve physical activity, gait, and balance in community-dwelling older adults [14-17]. However, the evidence on effective eHealth in geriatric rehabilitation is scarce and fragmented. To our knowledge, no prior reviews have examined the effectiveness of eHealth in geriatric rehabilitation.

To better understand how eHealth can be used safely, feasibility testing is an important first step $[18,19]$. The aim of feasibility testing is to "determine whether an intervention is appropriate for further testing" [20,21], but a general accepted standard on feasibility testing is lacking. Examples of factors that can be addressed in feasibility testing are adverse events, adherence, and acceptability [10].

Additionally, usable eHealth is also an important prerequisite for successful implementation [13,19,22]. Usability can be defined as "the extent to which a system, product, or service can be used by specified users to achieve specified goals with effectiveness, efficiency, and satisfaction in a specified context of use" [23]. For older adults receiving geriatric rehabilitation, usability is especially crucial, since there are certain age-related barriers that may hamper the usability of eHealth [24-26]. These barriers can be categorized into 4 patient-related domains: cognition, physical ability, perception, and motivation [27]. For example, poor vision can make it harder to distinguish certain icons on screens, or cognitive impairment might lead to problems understanding certain eHealth interventions. Often, eHealth is insufficiently tailored to these age-related barriers [28].

Therefore, a systematic review of eHealth in geriatric rehabilitation including the concepts feasibility, usability, and effectiveness was needed. This systematic review can help speed up the implementation process of eHealth and ensure successful adoption of eHealth overall. The aim of this review was to assess evidence on the effectiveness, feasibility, and usability of eHealth interventions in older adults in geriatric rehabilitation.

\section{Methods}

\section{Study Registration and Protocol}

This systematic review is registered at PROSPERO, with registration number CRD42019133192 [29]. This systematic review was based on the PRISMA (Preferred Reporting Items for Systematic Reviews and Meta-analyses) statement, which is an evidence-based minimum set of items used for reporting in systematic reviews and meta-analyses [30]. The complete checklist for this review can be found in Multimedia Appendix 1 .

\section{Types of Studies and Participants}

In this review, we included randomized controlled trials, nonrandomized studies, quantitative descriptive studies, qualitative research, and mixed methods studies. We excluded systematic reviews, abstracts, editorials, and non-English and nonpeer-reviewed studies. Studies were included that examined older patients with a mean age of $\geq 70$ years who received geriatric rehabilitation, which is in line with consensus statements on the organization and delivery of geriatric rehabilitation across Europe [31]. Because there is variability between countries' health care systems and consequently also between countries' provisions of geriatric rehabilitation [31,32], we included studies in different types of settings such as (geriatric) rehabilitation centers, skilled nursing facilities, hospitals, or ambulatory settings. Studies that included patients with a chronic disease with no acute functional decline were excluded.

\section{Interventions and Outcomes}

Studies investigated eHealth interventions applied during postacute geriatric rehabilitation. Outcome measures related to the effectiveness of interventions were included if they could be classified based on the World Health Organization's International Classification of Functioning, Disability, and Health (ICF) model [33], which covers the following domains: body functions and structure, activities, participation, environmental factors, and personal factors. For the purpose of this review, we chose to specify feasibility within the following domains: adverse events, adherence, and exclusion rates. Usability outcome measures were classified based on the MOLD-US framework, which is an evidence-based framework of aging barriers that influence the usability of eHealth in older adults and includes 4 categories: cognition, motivation, physical ability, and perception [27]. We included both primary and secondary outcome measures. 


\section{Sources and Search Strategy}

On March 9, 2019, March 10, 2019, and January 11, 2021, we searched the following databases: MEDLINE, PsycINFO, EMBASE, EMCARE, Cochrane Library, Web of Science, and Central databases. For this review, 3 separate search strings were compiled. The first focused on the effectiveness, the second focused on the feasibility, and the third focused on the usability of eHealth interventions in geriatric rehabilitation. The search string focusing on effectiveness included keywords related to older adults, rehabilitation, and eHealth interventions. Studies were identified when at least 2 of 3 keywords were present. The search strings focusing on feasibility and usability included an additional keyword related to feasibility or usability. In both search strings, keywords were combined using MeSH terms using the Boolean operations "or" and "and." The complete search strings can be found in Multimedia Appendix 2.

\section{Selection of Studies and Data Extraction}

We first screened titles of the identified studies. The abstracts of all potentially relevant studies were then screened by 2 authors independently. Next, full texts were obtained and reviewed by the same authors. We excluded studies that did not meet the inclusion criteria. Disagreements between the 2 authors were discussed until a consensus was reached. If a disagreement could not be resolved, a third reviewer was consulted. Data extraction was performed using Covidence, which is an online systematic review management tool [34]. In Covidence, a data extraction form was constructed that included details of publication (ie, author, year, title, country of study, and funding), study design, methods (ie, inclusion and exclusion criteria, population, randomization, statistical analysis, and outcome measures), sample characteristics (ie, age, number of participants, gender, and diagnosis), eHealth intervention (ie, name of intervention, goal of intervention, delivery of intervention, and application of intervention), and primary and secondary outcomes. As the complexity of eHealth interventions influences implementation, we sorted eHealth interventions ranging from simple (ie, video communications, health sensors, or gateways) to complex (ie, robotics, exergames, or virtual reality) $[9,35]$. One author then extracted the data. A subset of the data ( $10 \%$ of included studies) was also extracted by a second author to check interrater reliability.

\section{Quality Appraisal}

The quality of included studies was assessed using the Mixed Methods Appraisal Tool (MMAT) [36], which allowed quality assessment across different study designs. The MMAT is a critical appraisal tool specifically designed to assess the quality of 5 types of study designs: qualitative research, randomized controlled trials, nonrandomized studies, quantitative descriptive studies, and mixed methods studies. For each study design, the MMAT provides 5 quality criteria that must be rated with "Yes," "No," or "Can't tell." Since the calculation of an overall score from the ratings of each criterion is discouraged [36,37], we reported a separate score for each rating. Nevertheless, an overall score was reported, because it provides a general picture of study quality. Studies were not excluded based on study quality [36]. For the randomized controlled trials and nonrandomized designs, we rated the criterion "Are there complete outcome data?" as "No" when the drop-out rate was over $20 \%$ [38]. In nonrandomized designs, we rated the criterion "Are the confounders accounted for in the design and analysis?" as "No" when there was no description of additional therapy offered during the study, functional status, or cognitive status. Quality assessment was carried out by one author, and $10 \%$ of the included studies were selected at random and additionally assessed by a second author to check interrater reliability.

\section{Data Analysis and Data Synthesis}

In studies that reported outcomes related to effectiveness and included a control group, a narrative synthesis was conducted using a harvest plot [39]. In the harvest plot, primary and secondary outcomes were described and color coded based on ICF domain. For each study, the bars in the harvest plot indicated the total results for the different ICF domains, and the height of the bars represented the methodical quality based on the MMAT. When a study reported multiple consistent results within the same ICF domain, the results were combined in 1 bar. If a study reported conflicting results within the same ICF domain, both results were presented. Randomized controlled trails were represented by a thick contour around bars. A meta-analysis was not feasible since the included studies were too heterogeneous with regard to population, intervention, and outcome measures.

\section{Results}

\section{Study Selection}

The search strategy identified a total of 7635 unique records. After exclusion of records based on title and abstract, 331 records remained. During full-text screening, a further 291 records were excluded, resulting in the inclusion of 40 studies in this review. Reasons for exclusion are presented in the study flowchart shown in Figure 1. In 12 cases, a third reviewer was needed to achieve consensus during the process of study selection. 
Figure 1. PRISMA flow diagram of search strategy results. ICF: International Classification of Functioning, Disability, and Health.

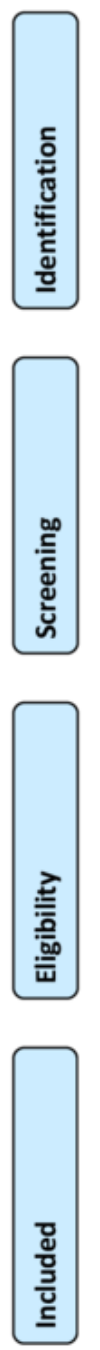

\section{Study Characteristics}

Study characteristics are shown in Table 1 . Of the 40 included studies, 18 (45\%) were randomized controlled trails [40-57], 2 $(5 \%)$ had a mixed methods design $[58,59], 1$ was a qualitative study [60], and $19(48 \%)$ had a quantitative nonrandomized design [61-79], of which 9 studies (of 19, 47\%) included a control group [53,61-68,79]. Of 40 studies, 17 studies (43\%) were conducted in a hospital setting $[41-44,46,50,51,55-57,62,64-66,68,71,79]$. Of the 17 hospital-setting studies, $12(71 \%)$ were conducted in a dedicated hospital-rehabilitation unit [41-44,46,50,51,55,56,64,71,79], 2 $(12 \%)$ were in a hospital-stroke unit $[57,68]$, and $1(6 \%)$ was conducted in a geriatric day hospital [62]. Of the 40 studies, 10 $(25 \%)$ were conducted in an ambulatory setting $[47,48,52-54,60,69,75,76,78], 9$ studies $(23 \%)$ took place in a geriatric rehabilitation setting [40,45,49,58,59,61,63,70,74], 2 studies $(5 \%)$ were at a tertiary rehabilitation center [60,73], 1 study (3\%) was at a skilled nursing facility [77], and 2 studies (5\%) did not report the setting $[67,72]$. 
Table 1. Study characteristics.

\begin{tabular}{|c|c|c|c|c|c|c|c|}
\hline $\begin{array}{l}\text { Author, year, } \\
\text { country }\end{array}$ & Design & $\begin{array}{l}\text { Diagnosis; n; set- } \\
\text { ting }\end{array}$ & $\begin{array}{l}\text { Age }(\mathrm{SD}) \\
\text { female }(\%)\end{array}$ & Intervention & Use of intervention & $\begin{array}{l}\text { Primary outcome } \\
\text { domain (primary } \\
\text { outcome measure) }\end{array}$ & $\begin{array}{l}\text { Secondary out- } \\
\text { come domain(s) }\end{array}$ \\
\hline $\begin{array}{l}\text { Barnason [53], } \\
\text { 2009, United } \\
\text { States }\end{array}$ & $\mathrm{RCT}^{\mathrm{a}}$ & $\begin{array}{l}\text { Cardiac; } \mathrm{n}=55 ; \\
\text { Ambulatory }\end{array}$ & $\begin{array}{l}71.6(5.1) \\
16\end{array}$ & $\begin{array}{l}\text { Video communi- } \\
\text { cation in combina- } \\
\text { tion with non- } \\
\text { eHealth vs usual } \\
\text { care }\end{array}$ & $\begin{array}{l}\text { Daily use, subjects re- } \\
\text { sponded to assessment } \\
\text { queries, were provided } \\
\text { with strategies }\end{array}$ & $\begin{array}{l}\text { Effectiveness, activi- } \\
\text { ties (other) }\end{array}$ & $\begin{array}{l}\text { Effectiveness, } \\
\text { participation }\end{array}$ \\
\hline $\begin{array}{l}\text { Backman [59], } \\
\text { 2020, United } \\
\text { Kingdom }\end{array}$ & $\begin{array}{l}\text { Mixed meth- } \\
\text { ods }\end{array}$ & $\begin{array}{l}\text { Orthopedic; } \\
\mathrm{n}=30 ; \text { Geriatric } \\
\text { rehabilitation }\end{array}$ & $\begin{array}{l}81(67-96) ; \\
63\end{array}$ & Mobile apps & $\begin{array}{l}\text { Providing access to dis- } \\
\text { charge records during } \\
\text { transition to home }\end{array}$ & Usability & $-\mathrm{b}$ \\
\hline $\begin{array}{l}\text { Bernocchi } \\
\text { [52], 2018, } \\
\text { Italy }\end{array}$ & RCT & $\begin{array}{l}\text { Multiple diag- } \\
\text { noses; } n=146 ; \\
\text { Ambulatory }\end{array}$ & $\begin{array}{l}79(6.5) \\
84\end{array}$ & $\begin{array}{l}\text { Video communi- } \\
\text { cation in combina- } \\
\text { tion with non- } \\
\text { eHealth vs usual } \\
\text { care }\end{array}$ & $\begin{array}{l}\text { Weekly calls; video } \\
\text { communication } \\
2 \times / \text { month; fall preven- } \\
\text { tion program provided } \\
\text { by therapist }\end{array}$ & $\begin{array}{l}\text { Effectiveness, activi- } \\
\text { ties (other) }\end{array}$ & $\begin{array}{l}\text { Feasibility, ef- } \\
\text { fectiveness, ac- } \\
\text { tivities, partici- } \\
\text { pation }\end{array}$ \\
\hline $\begin{array}{l}\text { Bernocchi } \\
\text { [69], 2016, } \\
\text { Italy }\end{array}$ & $\begin{array}{l}\text { Quantitative; } \\
\text { nonrandom- } \\
\text { ized }\end{array}$ & $\begin{array}{l}\text { Stroke; } \mathrm{n}=15 \\
\text { Ambulatory }\end{array}$ & $71(11) ; 47$ & $\begin{array}{l}\text { Video communi- } \\
\text { cation in combina- } \\
\text { tion with health } \\
\text { sensors }\end{array}$ & $\begin{array}{l}\text { Weekly calls with } \\
\text { nurse; weekly video } \\
\text { communication with } \\
\text { physiotherapist }\end{array}$ & $\begin{array}{l}\text { Feasibility (n com- } \\
\text { pleted, n sessions) }\end{array}$ & $\begin{array}{l}\text { Effectiveness, } \\
\text { body functions, } \\
\text { activities }\end{array}$ \\
\hline $\begin{array}{l}\text { Cannell [44], } \\
\text { 2017, Aus- } \\
\text { tralia }\end{array}$ & RCT & $\begin{array}{l}\text { Stroke; } \mathrm{n}=40 ; \\
\text { Hospital, rehabili- } \\
\text { tation unit }\end{array}$ & $\begin{array}{l}74(10) \\
37.5\end{array}$ & $\begin{array}{l}\text { Exergames in } \\
\text { combination with } \\
\text { virtual reality vs } \\
\text { usual care }\end{array}$ & $\begin{array}{l}1 \text { hour/session, } 5 \\
\text { days/week, in addition } \\
\text { to conventional therapy }\end{array}$ & $\begin{array}{l}\text { Effectiveness, activi- } \\
\text { ties (maintaining } \\
\text { body position) }\end{array}$ & $\begin{array}{l}\text { Effectiveness, } \\
\text { activities }\end{array}$ \\
\hline $\begin{array}{l}\text { Chan [62], } \\
\text { 2012, China }\end{array}$ & $\begin{array}{l}\text { Quantitative } \\
\text { nonrandom- } \\
\text { ized }\end{array}$ & $\begin{array}{l}\text { Multiple diag- } \\
\text { noses; n=90; } \\
\text { Geriatric Day } \\
\text { hospital }\end{array}$ & $\begin{array}{l}80(7.1) \\
73\end{array}$ & $\begin{array}{l}\text { Exergames vs } \\
\text { usual care }\end{array}$ & $\begin{array}{l}10 \mathrm{~min} / \mathrm{session}, 8 \text { ses- } \\
\text { sions total, in addition } \\
\text { to conventional therapy }\end{array}$ & $\begin{array}{l}\text { Feasibility (total } \\
\text { time spent, average } \\
\mathrm{BS}^{\mathrm{c}} \text { and } \% \mathrm{MHR}^{\mathrm{d}} \text { ) }\end{array}$ & $\begin{array}{l}\text { Effectiveness, } \\
\text { activities }\end{array}$ \\
\hline $\begin{array}{l}\text { Cimarolli } \\
\text { [77], 2017, } \\
\text { United States }\end{array}$ & $\begin{array}{l}\text { Quantitative; } \\
\text { nonrandom- } \\
\text { ized }\end{array}$ & $\begin{array}{l}\text { Multiple diag- } \\
\text { noses; } n=237 ; \\
\text { Skilled nurse fa- } \\
\text { cility }\end{array}$ & $\begin{array}{l}76(10.7) \\
59\end{array}$ & Exergames & $\begin{array}{l}\text { Recommended use: } 2 \\
\text { sessions/week for } 15 \\
\text { min, in addition to con- } \\
\text { ventional therapy }\end{array}$ & $\begin{array}{l}\text { Feasibility (time } \\
\text { spent, predictors of } \\
\text { intense use) }\end{array}$ & $\begin{array}{l}\text { Effectiveness, } \\
\text { external factors }\end{array}$ \\
\hline $\begin{array}{l}\text { Dakin [61], } \\
\text { 2011, Aus- } \\
\text { tralia }\end{array}$ & $\begin{array}{l}\text { Quantitative; } \\
\text { nonrandom- } \\
\text { ized }\end{array}$ & $\begin{array}{l}\text { Multiple diag- } \\
\text { noses; } n=34 ; \\
\text { Geriatric rehabili- } \\
\text { tation }\end{array}$ & $77 ; 47$ & $\begin{array}{l}\text { Health sensors vs } \\
\text { usual care }\end{array}$ & $\begin{array}{l}\text { Wore health sensor dai- } \\
\text { ly during admission }\end{array}$ & $\begin{array}{l}\text { Effectiveness activi- } \\
\text { ties }\left(\mathrm{ADL}^{\mathrm{e}}\right)\end{array}$ & $\begin{array}{l}\text { Effectiveness, } \\
\text { external factors }\end{array}$ \\
\hline $\begin{array}{l}\text { Da-Silva [57], } \\
\text { 2019, United } \\
\text { Kingdom }\end{array}$ & $\mathrm{RCT}$ & $\begin{array}{l}\text { Stroke; } \mathrm{n}=33 ; \\
\text { Hospital, stroke } \\
\text { unit }\end{array}$ & $71 ; 60.6$ & $\begin{array}{l}\text { Health sensors } \\
\text { with reminders vs } \\
\text { health sensors } \\
\text { without re- } \\
\text { minders }\end{array}$ & $\begin{array}{l}\text { Wore health sensor for } \\
4 \text { weeks, health sensor } \\
\text { vibrated to remind pa- } \\
\text { tients to use affected } \\
\text { arm }\end{array}$ & $\begin{array}{l}\text { Effectiveness, activi- } \\
\text { ties (hand and arm } \\
\text { use) }\end{array}$ & $\begin{array}{l}\text { Feasibility, ad- } \\
\text { herence }\end{array}$ \\
\hline $\begin{array}{l}\text { Doornebosch } \\
{[70], 2007 \text {, }} \\
\text { Netherlands }\end{array}$ & $\begin{array}{l}\text { Quantitative; } \\
\text { nonrandom- } \\
\text { ized }\end{array}$ & $\begin{array}{l}\text { Stroke; } n=10 ; \\
\text { Geriatric rehabili- } \\
\text { tation }\end{array}$ & $\begin{array}{l}72(53-94) \\
80\end{array}$ & Robotics & $\begin{array}{l}20 \text { minutes/session, } 8 \\
\text { sessions total, in addi- } \\
\text { tion to conventional } \\
\text { therapy }\end{array}$ & $\begin{array}{l}\text { Personal factors (pa- } \\
\text { tient's experience) }\end{array}$ & $\begin{array}{l}\text { Effectiveness, } \\
\text { body functions }\end{array}$ \\
\hline $\begin{array}{l}\text { Edmans [68], } \\
\text { 2009, United } \\
\text { Kingdom }\end{array}$ & $\begin{array}{l}\text { Quantitative; } \\
\text { nonrandom- } \\
\text { ized }\end{array}$ & $\begin{array}{l}\text { Stroke; } \mathrm{n}=13 ; \\
\text { Hospital, stroke } \\
\text { unit }\end{array}$ & $73 ; 23$ & $\begin{array}{l}\text { Virtual reality vs } \\
\text { usual care }\end{array}$ & $\begin{array}{l}1 \text { hour/session, } 5 \\
\text { days/week }\end{array}$ & $\begin{array}{l}\text { Effectiveness, activi- } \\
\text { ties (other) }\end{array}$ & $\begin{array}{l}\text { Effectiveness, } \\
\text { activities }\end{array}$ \\
\hline $\begin{array}{l}\text { Franceschini } \\
{[56], 2020,} \\
\text { Italy }\end{array}$ & $\mathrm{RCT}$ & $\begin{array}{l}\text { Stroke; } \mathrm{n}=48 ; \\
\text { Hospital, rehabili- } \\
\text { tation unit }\end{array}$ & $\begin{array}{l}72(64.3) \\
45.8\end{array}$ & $\begin{array}{l}\text { Robotics vs usual } \\
\text { care }\end{array}$ & $\begin{array}{l}30 \text { minutes/session, } 5 \\
\text { days/week over } 6 \\
\text { weeks, in addition to } \\
\text { conventional therapy }\end{array}$ & $\begin{array}{l}\text { Effectiveness, body } \\
\text { functions (muscle } \\
\text { power, tone, and re- } \\
\text { flexes) }\end{array}$ & $\begin{array}{l}\text { Effectiveness, } \\
\text { (muscle power, } \\
\text { tone, and reflex- } \\
\text { es) }\end{array}$ \\
\hline $\begin{array}{l}\text { Gandolfi [71], } \\
\text { 2017, Italy }\end{array}$ & $\begin{array}{l}\text { Quantitative; } \\
\text { nonrandom- } \\
\text { ized }\end{array}$ & $\begin{array}{l}\text { Stroke; } \mathrm{n}=2 ; \text { Hos- } \\
\text { pital, rehabilita- } \\
\text { tion unit }\end{array}$ & $74 ; 100$ & Robotics & $\begin{array}{l}20 \text { minutes/session, } 5 \\
\text { days/week, } 10 \text { sessions } \\
\text { total, in addition to } \\
\text { conventional therapy }\end{array}$ & $\begin{array}{l}\text { Feasibility (compli- } \\
\text { ance, time to set de- } \\
\text { vice) }\end{array}$ & $\begin{array}{l}\text { Effectiveness, } \\
\text { body functions }\end{array}$ \\
\hline $\begin{array}{l}\text { Goto [65], } \\
\text { 2017, Japan }\end{array}$ & $\begin{array}{l}\text { Quantitative; } \\
\text { nonrandom- } \\
\text { ized }\end{array}$ & $\begin{array}{l}\text { Orthopedic; } \\
\mathrm{n}=20 ; \text { Hospital }\end{array}$ & $\begin{array}{l}74(7.5) \\
90\end{array}$ & $\begin{array}{l}\text { Robotics vs usual } \\
\text { care }\end{array}$ & $\begin{array}{l}\text { Every other day, in addi- } \\
\text { tion to conventional } \\
\text { therapy }\end{array}$ & $\begin{array}{l}\text { Effectiveness, body } \\
\text { functions (mobility } \\
\text { of joints) }\end{array}$ & $\begin{array}{l}\text { Effectiveness, } \\
\text { body functions }\end{array}$ \\
\hline
\end{tabular}




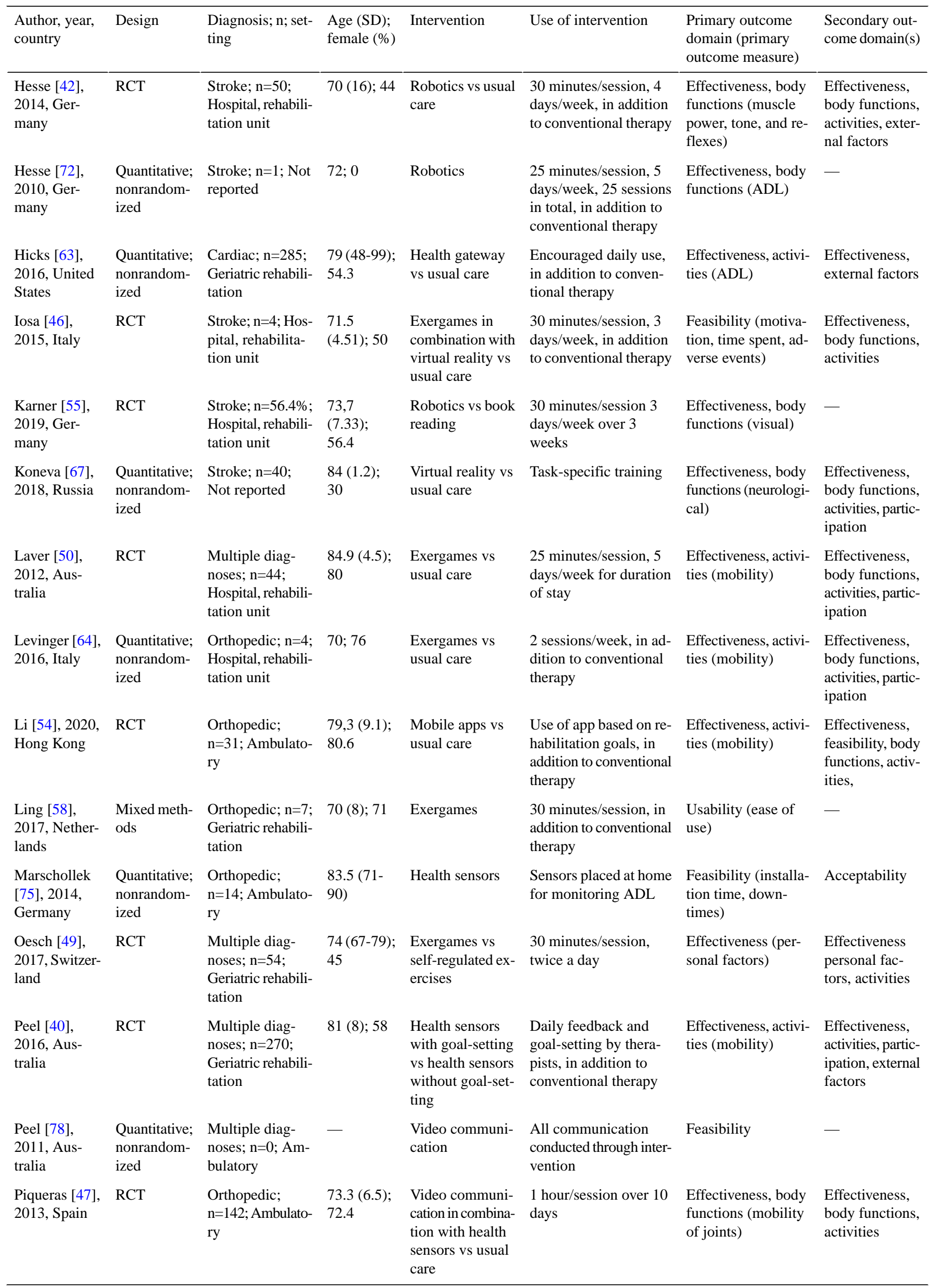




\begin{tabular}{|c|c|c|c|c|c|c|c|}
\hline $\begin{array}{l}\text { Author, year, } \\
\text { country }\end{array}$ & Design & $\begin{array}{l}\text { Diagnosis; n; set- } \\
\text { ting }\end{array}$ & $\begin{array}{l}\text { Age (SD); } \\
\text { female }(\%)\end{array}$ & Intervention & Use of intervention & $\begin{array}{l}\text { Primary outcome } \\
\text { domain (primary } \\
\text { outcome measure) }\end{array}$ & $\begin{array}{l}\text { Secondary out- } \\
\text { come domain(s) }\end{array}$ \\
\hline $\begin{array}{l}\text { Sampson [73], } \\
\text { 2012, New } \\
\text { Zealand }\end{array}$ & $\begin{array}{l}\text { Quantitative; } \\
\text { nonrandom- } \\
\text { ized }\end{array}$ & $\begin{array}{l}\text { Stroke; } \mathrm{n}=1 ; \mathrm{Re}- \\
\text { habilitation cen- } \\
\text { ter }\end{array}$ & $76 ; 100$ & $\begin{array}{l}\text { Robotics in com- } \\
\text { bination with vir- } \\
\text { tual reality }\end{array}$ & $\begin{array}{l}45 \text { minutes/session, } 4 \\
\text { sessions/week over } 6 \\
\text { weeks, in addition to } \\
\text { conventional therapy }\end{array}$ & $\begin{array}{l}\text { Effectiveness, body } \\
\text { functions (muscle } \\
\text { power, tone, and re- } \\
\text { flexes) }\end{array}$ & $\begin{array}{l}\text { Effectiveness } \\
\text { body functions }\end{array}$ \\
\hline $\begin{array}{l}\text { Schoone [45], } \\
\text { 2011, Nether- } \\
\text { lands }\end{array}$ & $\mathrm{RCT}$ & $\begin{array}{l}\text { Stroke; } n=24 ; \\
\text { Geriatric rehabili- } \\
\text { tation }\end{array}$ & $\begin{array}{l}71.3(8.2) \\
33\end{array}$ & Robotics & $\begin{array}{l}10-30 \text { minutes/sessions, } \\
3 \text { sessions/week over } 6 \\
\text { weeks, in addition to } \\
\text { conventional therapy }\end{array}$ & $\begin{array}{l}\text { Effectiveness, body } \\
\text { functions, activities } \\
\text { (hand and arm use) }\end{array}$ & $\begin{array}{l}\text { Effectiveness } \\
\text { participation, } \\
\text { external factors }\end{array}$ \\
\hline $\begin{array}{l}\text { Schwickert } \\
\text { [74], 2011, } \\
\text { Germany }\end{array}$ & $\begin{array}{l}\text { Quantitative; } \\
\text { nonrandom- } \\
\text { ized }\end{array}$ & $\begin{array}{l}\text { Orthopedic; } n=8 ; \\
\text { Geriatric rehabili- } \\
\text { tation }\end{array}$ & $79.5 ; 50$ & $\begin{array}{l}\text { Robotics, virtual } \\
\text { reality }\end{array}$ & $\begin{array}{l}\text { 30-45 minutes/session, } \\
\text { 2-3 sessions/week for } \\
\text { 2-4 weeks, in addition } \\
\text { to conventional therapy }\end{array}$ & $\begin{array}{l}\text { Feasibility (adher- } \\
\text { ence, satisfaction) }\end{array}$ & $\begin{array}{l}\text { Effectiveness, } \\
\text { body functions, } \\
\text { activities, partic- } \\
\text { ipation }\end{array}$ \\
\hline $\begin{array}{l}\text { Takano [79], } \\
\text { 2020, Japan }\end{array}$ & $\begin{array}{l}\text { Quantitative; } \\
\text { nonrandom- } \\
\text { ized }\end{array}$ & $\begin{array}{l}\text { Orthopedic; } \\
\mathrm{n}=27 ; \text { Hospital, } \\
\text { rehabilitation unit }\end{array}$ & $\begin{array}{l}81(6.3) \\
89\end{array}$ & $\begin{array}{l}\text { Robotics in com- } \\
\text { bination with ex- } \\
\text { ergames }\end{array}$ & $\begin{array}{l}20 \mathrm{~min} / \mathrm{session} 6 \text { ses- } \\
\text { sions/week for } 2 \text { weeks } \\
\text { in addition to conven- } \\
\text { tional therapy }\end{array}$ & $\begin{array}{l}\text { Effectiveness activi- } \\
\text { ties (mobility) }\end{array}$ & $\begin{array}{l}\text { Effectiveness, } \\
\text { activities, }\end{array}$ \\
\hline $\begin{array}{l}\text { Taveggia [43], } \\
\text { 2016, Italy }\end{array}$ & RCT & $\begin{array}{l}\text { Stroke; } \mathrm{n}=28 ; \\
\text { Hospital, rehabili- } \\
\text { tation unit }\end{array}$ & $72(6) ; 39$ & $\begin{array}{l}\text { Robotics vs usual } \\
\text { care }\end{array}$ & $\begin{array}{l}30 \text { minutes/session, } 5 \\
\text { sessions/week over } 5 \\
\text { weeks, in addition to } \\
\text { conventional therapy }\end{array}$ & $\begin{array}{l}\text { Effectiveness, activi- } \\
\text { ties (mobility) }\end{array}$ & $\begin{array}{l}\text { Effectiveness, } \\
\text { activities, partic- } \\
\text { ipation }\end{array}$ \\
\hline $\begin{array}{l}\text { Van den Berg } \\
{[51], 2015,} \\
\text { Australia }\end{array}$ & RCT & $\begin{array}{l}\text { Multiple diag- } \\
\text { noses; } n=58 ; \\
\text { Hospital, rehabili- } \\
\text { tation unit }\end{array}$ & $80(12) ; 62$ & $\begin{array}{l}\text { Exergames vs } \\
\text { usual care }\end{array}$ & $\begin{array}{l}1 \text { hour/session, } 5 \text { ses- } \\
\text { sion/week, in addition } \\
\text { to conventional therapy }\end{array}$ & $\begin{array}{l}\text { Effectiveness, activi- } \\
\text { ties (mobility) }\end{array}$ & $\begin{array}{l}\text { Usability; Effec- } \\
\text { tiveness, activi- } \\
\text { ties, participa- } \\
\text { tion }\end{array}$ \\
\hline $\begin{array}{l}\text { Vanoglio [41], } \\
2017, \text { Italy }\end{array}$ & RCT & $\begin{array}{l}\text { Stroke; } \mathrm{n}=30 ; \\
\text { Hospital, rehabili- } \\
\text { tation unit }\end{array}$ & $71(12) ; 53$ & $\begin{array}{l}\text { Robotics vs usual } \\
\text { care }\end{array}$ & $\begin{array}{l}40 \text { minutes/session, } 5 \\
\text { sessions/week over } 6 \\
\text { weeks }\end{array}$ & $\begin{array}{l}\text { Feasibility (n com- } \\
\text { pleted, adverse } \\
\text { events, difficulty) }\end{array}$ & $\begin{array}{l}\text { Effectiveness, } \\
\text { body functions, } \\
\text { external factors }\end{array}$ \\
\hline $\begin{array}{l}\text { White [60], } \\
\text { 2015, Aus- } \\
\text { tralia }\end{array}$ & Qualitative & $\begin{array}{l}\text { Stroke; } \mathrm{N}=12 ; \\
\text { Rehabilitation } \\
\text { center, ambulato- } \\
\text { ry }\end{array}$ & $\begin{array}{l}73(53-83) \\
33\end{array}$ & Mobile apps & $\begin{array}{l}\text { Therapist installed } \\
\text { apps; patients encour- } \\
\text { aged to explore iPad }\end{array}$ & Usability & - \\
\hline $\begin{array}{l}\text { Yoshikawa } \\
\text { [66], 2018, } \\
\text { Japan }\end{array}$ & $\begin{array}{l}\text { Quantitative; } \\
\text { nonrandom- } \\
\text { ized }\end{array}$ & $\begin{array}{l}\text { Orthopedic; } \\
\mathrm{n}=19 ; \text { Hospital }\end{array}$ & $\begin{array}{l}76(6.85) \\
81\end{array}$ & $\begin{array}{l}\text { Robotics vs usual } \\
\text { care }\end{array}$ & $\begin{array}{l}14 \text { minutes/session, } 12- \\
14 \text { session in } 4 \text { weeks, } \\
\text { in addition to conven- } \\
\text { tional therapy }\end{array}$ & $\begin{array}{l}\text { Effectiveness, activi- } \\
\text { ties (mobility) }\end{array}$ & $\begin{array}{l}\text { Effectiveness, } \\
\text { body functions }\end{array}$ \\
\hline
\end{tabular}

${ }^{\mathrm{a}} \mathrm{RCT}$ : randomized controlled trial.

${ }^{\mathrm{b}}$ Not available.

${ }^{\mathrm{c}}$ BS: Borg Perceived Exertion Scale.

$\mathrm{d}_{\% \mathrm{MHR}}$ : maximum heart rate.

e ADL: activities of daily living.

Of 40 studies, 17 (43\%) included participants who were diagnosed with stroke [41-46,55-57,60,67-73], 10 (25\%) included participants with multiple diagnoses [40,49-52,59,61,62,76-78], 11 (28\%) included participants with orthopedic problems [47,48,54,58,59,64-66,74,75,79], and 2 studies $(5 \%)$ included participants with cardiac-related diagnoses
[53,63]. Across all studies, the included sample size ranged from 1 to 285 participants.

Various types of eHealth interventions were used. Of 40 studies, 11 studies $(28 \%)$ delivered the intervention via robotics [41-43,45,55,56,65,66,70-72], 2 studies (5\%) combined robotics with virtual reality $[73,74]$, and 1 study $(3 \%)$ combined robotics 
with exergames [79]. Additionally, 9 studies (of 40, 23\%) investigated exergames [44,46,49-51,58,62,64,77], of which 2 (of $9,22 \%$ ) combined exergames with virtual reality $[44,46]$ and 1 (of $9,11 \%$ ) combined exergames with health sensors [51]. Of 40 studies, 2 (5\%) examined video communication [76,78], $3(8 \%)$ combined video communication with health sensors [47,53,69], and $1(3 \%)$ combined video communication with a non-eHealth intervention [52]. Of 40 studies, health sensors were used in 6 studies $(15 \%)$ [40,48,57,61,63,75], including 1 (of $6,17 \%$ ) in combination with a health gateway [63] and 1 (of $6,17 \%$ ) in combination with a non-eHealth intervention [48]. Of 40 studies, 3 studies (8\%) investigated mobile apps $[54,59,60]$, and 2 studies $(5 \%)$ examined virtual reality $[67,68]$.

Outcome measures related to effectiveness were reported in 24 of 40 studies (60\%) [40,42-45,47-50,53,55,56,61,63-68, $70,72,73,76,79]$, and 10 of 40 studies $(25 \%)$ included outcome measures related to effectiveness and feasibility $[41,46,52,54,57,62,69,71,74,77]$. Of 40 studies, 2 studies (5\%) included outcomes related to usability [58,60], 2 studies $(5 \%)$ included outcomes related only to feasibility [75,78], 1 study (3\%) included outcomes related to effectiveness and usability
[51], and 1 study (3\%) included outcomes related to feasibility and usability [59]. A detailed description of all included studies can be found in Multimedia Appendix 3.

\section{Study Quality}

Results of the quality assessment are presented in Figure 2 and Figure 3 . The quality of the included studies ranged from -3 to 5 (on a scale ranging from -5 to 5 ). The mean overall score was 3 for randomized controlled trails, 1 for quantitative nonrandomized studies, 1 for a mixed methods studies, and 5 for a qualitative study (based on 1 study). In quantitative nonrandomized studies, the most frequent shortcoming was insufficient reporting of confounders; only 2 of 19 studies (11\%) accounted for confounders in design and analysis [73,79]. The representativeness of the target population in quantitative nonrandomized studies was also often insufficient; 9 of the 19 studies $(47 \%)$ reported insufficient information, lacking either adequate explanation of why certain eligible participants chose not to participate or a clear description of the target population $[53,61,65,67,69,71,75,76,78]$. Additionally, 6 of the 19 studies $(32 \%)$ included a sample size of less than 20 [64,66,70,72-74].

Figure 2. Quality appraisal for randomized controlled trial studies.

\begin{tabular}{|c|c|c|c|c|c|c|}
\hline $\begin{array}{l}\text { Randomized controlled trails } \\
\text { studies }\end{array}$ & $\begin{array}{l}\text { Randomization } \\
\text { appropriately } \\
\text { performed? }\end{array}$ & $\begin{array}{c}\text { Groups } \\
\text { comparable? }\end{array}$ & $\begin{array}{c}\text { Complete } \\
\text { outcome data? }\end{array}$ & $\begin{array}{l}\text { Assessors } \\
\text { blinded? }\end{array}$ & $\begin{array}{c}\text { Adhere to } \\
\text { intervention? }\end{array}$ & $\begin{array}{l}\text { Overall } \\
\text { score }\end{array}$ \\
\hline Vanoglio, et al (2017) [41] & & & & & & \\
\hline Hesse, et al (2014) [42] & & & & & & \\
\hline Taveggia, et al (2016) [43] & & & & & & \\
\hline Cannell, et al (2018) [44] & & & & & & \\
\hline Schoone, et al (2011) [45] & & & & & & \\
\hline Piqueras, et al (2013) [47] & & & & & & \\
\hline Pol, et al (2019) [48] & & & & & & \\
\hline Oesch, et al (2017) [49] & & & & & & \\
\hline Peel, et al (2016) [40] & & & & & & \\
\hline Laver, et al (2012) [50] & & & & & & \\
\hline Berg, et al (2016) [51] & & & & & & \\
\hline losa, et al (2010) [46] & & & & & & 1 \\
\hline Barnason, et al (2009) [53] & & & & & & 1 \\
\hline Bernocchi, et al (2018) [52] & & & & & & \\
\hline Franceschini, et al (2019) [56] & & & & & & \\
\hline $\mathrm{Li}$, et al (2020) [54] & & & & & & 2 \\
\hline Karner, et al (2019) [55] & & & & & & \\
\hline Da-Silva, et al (2019) [57] & & & & & & \\
\hline
\end{tabular}

Score Calculation

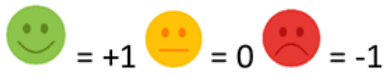


Figure 3. Quality appraisal for quantitative nonrandomized, qualitative, and mixed methods studies.

\begin{tabular}{|c|c|c|c|c|c|c|}
\hline $\begin{array}{l}\text { Quantitative nonrandomized } \\
\text { studies }\end{array}$ & $\begin{array}{c}\text { Representative } \\
\text { of the target } \\
\text { population? }\end{array}$ & $\begin{array}{c}\text { Are the } \\
\text { measurements } \\
\text { appropriate? }\end{array}$ & $\begin{array}{c}\text { Complete } \\
\text { outcome } \\
\text { data? }\end{array}$ & $\begin{array}{l}\text { Confounders } \\
\text { accounted for? }\end{array}$ & $\begin{array}{l}\text { Intervention } \\
\text { administered } \\
\text { as intended? }\end{array}$ & $\begin{array}{c}\text { Overall } \\
\text { score }\end{array}$ \\
\hline Edmans, et al (2009) [68] & & & & & & \\
\hline Koneva, et al (2018) [67] & & & & & & \\
\hline Yoshikawa, et al (2018) [66] & & & & & & \\
\hline Goto, et al (2017) [65] & & & & & & \\
\hline Levinger, et al (2016) [64] & & & & & & \\
\hline Hicks, et al (2016) [63] & & & & & & \\
\hline Chan, et al (2012) [62] & & & & & & \\
\hline Dakin, et al (2011) [61] & & & & & & \\
\hline Bernocchi, et al (2016) [69] & & & & & & \\
\hline Doornebosch,(2007) [70] & & & & & & \\
\hline Gandolfi, et al (2017) [71] & & & & & & \\
\hline Hesse, et al (2010) [72] & & & & & & \\
\hline Sampson, et al (2012) [73] & & & & & & \\
\hline Schwickert, et al (2011) [74] & & & & & & \\
\hline Marschollek, (2014) [75] & & & & & & \\
\hline Tousignant, et al (2006) [76] & & & & & & \\
\hline Cimarolli, et al (2017) [77] & & & & & & \\
\hline Peel, et al (2011) [78] & & & & & & \\
\hline Takano, et al (2020) [79] & & & & & & \\
\hline Qualitative studies & $\begin{array}{c}\text { Qualitative } \\
\text { approach } \\
\text { appropriate? }\end{array}$ & $\begin{array}{c}\text { Data collection } \\
\text { methods } \\
\text { adequate? }\end{array}$ & $\begin{array}{l}\text { Findings } \\
\text { adequately } \\
\text { derived from } \\
\text { the data? }\end{array}$ & $\begin{array}{c}\text { Results } \\
\text { interpreted } \\
\text { sufficiently by } \\
\text { data? }\end{array}$ & $\begin{array}{l}\text { Coherence in } \\
\text { data, analysis, } \\
\text { and } \\
\text { interpretation? }\end{array}$ & $\begin{array}{l}\text { Overall } \\
\text { score }\end{array}$ \\
\hline White, et al (2015) [60] & & & & & & \\
\hline Mixed methods studies & $\begin{array}{c}\text { Adequate } \\
\text { rationale for } \\
\text { mixed methods } \\
\text { design? }\end{array}$ & $\begin{array}{l}\text { Different } \\
\text { components } \\
\text { integrated? }\end{array}$ & $\begin{array}{c}\text { Integration of } \\
\text { qualitative } \\
\text { and } \\
\text { quantitative? }\end{array}$ & $\begin{array}{l}\text { Divergences or } \\
\text { inconsistencies } \\
\text { addressed? }\end{array}$ & $\begin{array}{l}\text { Components of } \\
\text { study adhere } \\
\text { to quality } \\
\text { criteria? }\end{array}$ & $\begin{array}{c}\text { Overall } \\
\text { score }\end{array}$ \\
\hline Ling, $Y$, et al (2017) [58] & & & & & & 1 \\
\hline Backman, et al (2020) [59] & & & & & & \\
\hline
\end{tabular}

\section{Effectiveness}

\section{Main Results for Effectiveness}

Across all studies with a control group ( $\mathrm{n}=27 ; 27 / 40,68 \%), 73$ different outcome measures were reported that were related to effectiveness, including 16 (22\%) within the ICF domain "body functions," $40(55 \%)$ in the domain "activities," $11(15 \%)$ in the domain "participation," $4(5 \%)$ in the domain "external factors," and 2 (3\%) in the domain "personal factors" (Figure
4). In 15 studies (of 27, 56\%), eHealth interventions were found to be at least as effective as non-eHealth interventions when focusing on the primary outcome measure, and 11 studies (of $27,41 \%$ ) reported eHealth interventions to be more effective than non-eHealth interventions. Of 27 studies, 1 study (4\%) reported beneficial outcomes in favor of the non-eHealth interventions. Results for each ICF domain are described in detail below. A harvest plot illustrating the evidence regarding effectiveness is presented in Figure 5. 
Figure 4. Outcome measures classified by the International Classification of Functioning, Disability, and Health model.

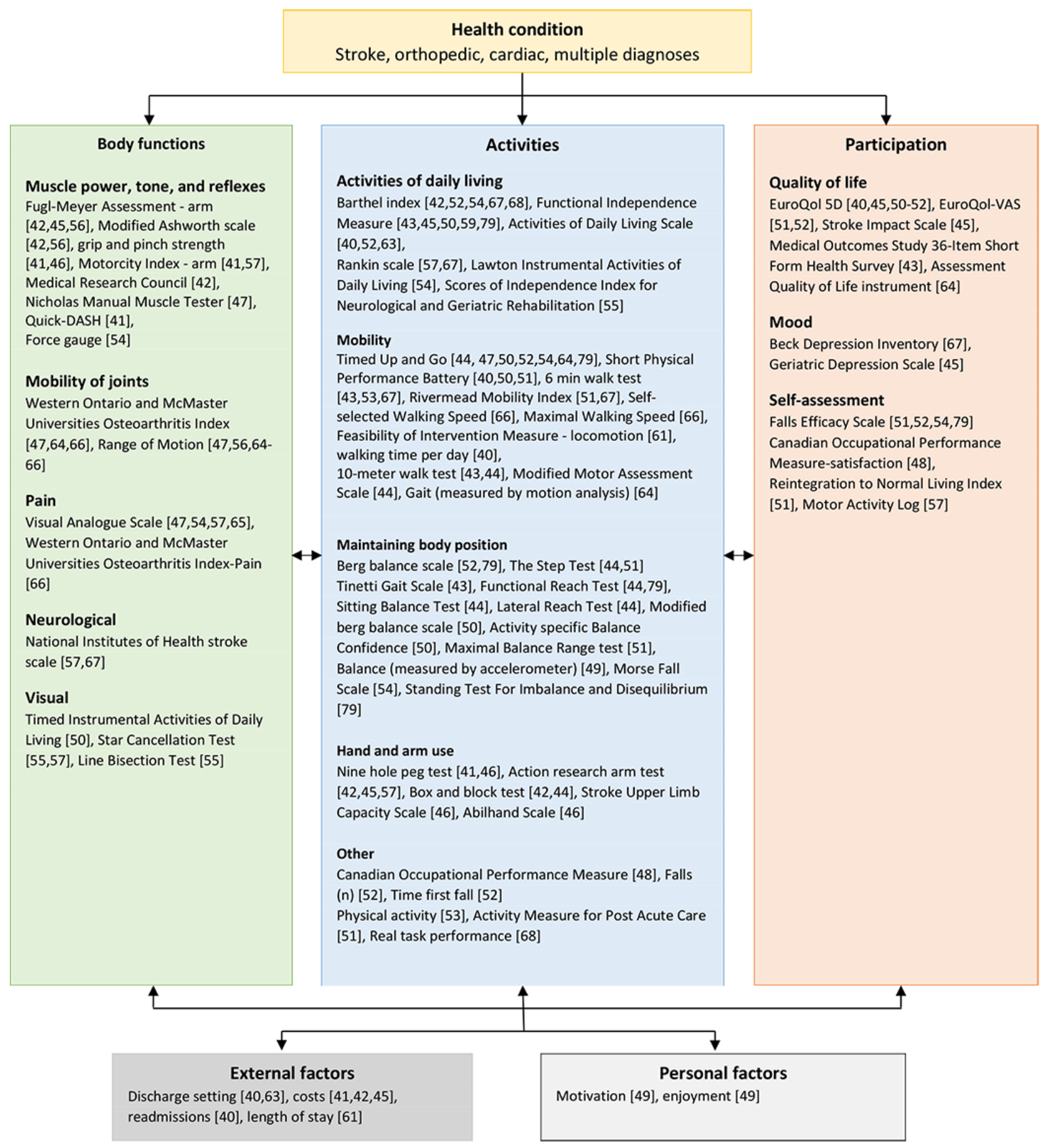


Figure 5. Harvest plot: effectiveness of eHealth interventions. MMAT: Mixed Methods Appraisal Tool.

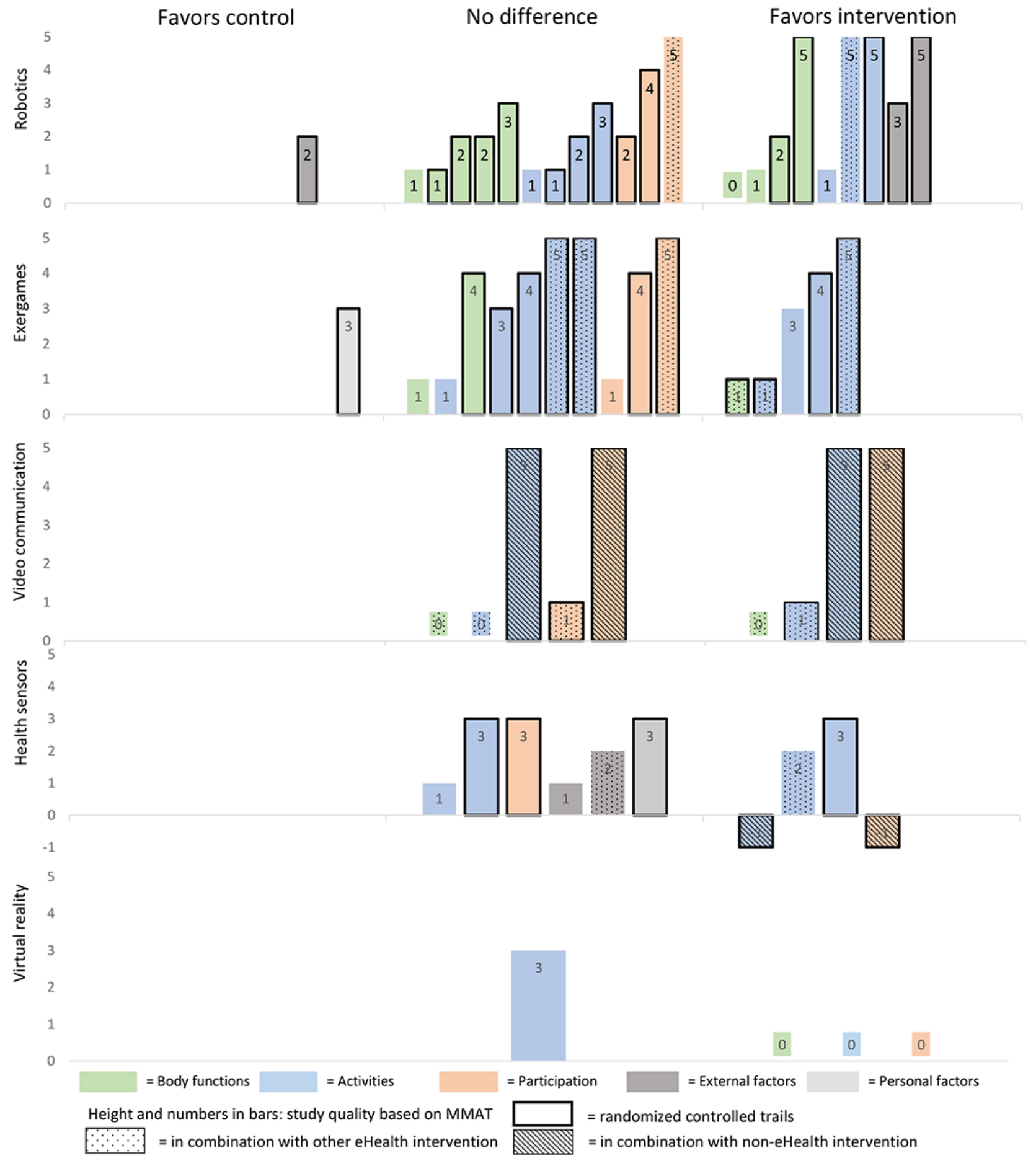

\section{Body Functions}

Of 40 total studies, 14 studies $(35 \%)$ included 16 outcomes related to body functions [41,42,45-47,50,54-57,64-67]. Of these 14 studies, 9 studies ( $64 \%$ ) found, in 7 outcome measures, significant improvements in favor of the intervention group (Figure 5) [41,46,47,54-56,65-67]. Of 14 studies, 4 studies (29\%) reported improved muscle power through robotics [56,65], exergames [46], or mobile apps [54]. Of 14 studies, 4 studies $(29 \%)$ found that the addition of robotics $[56,65,66]$ or video communication in combination with health sensors [47] improved the mobility of joints when compared with physical

therapy alone. Another 2 studies (of 14, 14\%) reported that the use of robotics could decrease pain when compared with conventional physiotherapy $[65,66]$. Koneva and colleagues [67] reported that the use of virtual reality improved neurological status, as measured by the National Institutes of Health stroke scale, when compared with usual care $(5.2 \pm 0.4$ vs $6.3 \pm 0.5$; $P<.001)$.

\section{Activities}

Of all 40 included studies, 25 studies $(63 \%)$ reported 40 outcomes related to activities [40-55,57,61-64,66-68,79], and 13 studies (33\%) found, in 17 outcomes, a significant outcome 
in favor of the intervention group [40,41,46,48,50-53,62,63,66,67,79]. Of 40 studies, 5 studies $(13 \%)$ demonstrated that eHealth was effective in improving activities of daily living when the intervention was delivered via video communication in combination with health sensors and a non-eHealth intervention [52] or when the intervention was delivered via health sensors in combination with health gateways [63], exergames [62], robotics [79], or virtual reality [67]. In these studies, eHealth was compared with usual care $[52,67]$, physiotherapy [62,79], or no intervention [63]. Another 6 studies (of 40,15\%) found that eHealth could contribute to improved mobility through the use of robotics [52,79], exergames [50], virtual reality [67], video communication in combination with health sensors [52], or health sensors in combination with goal setting [40]. These interventions were compared with physiotherapy [50,66,79], usual care [52,67], or health sensors without goal setting [40]. Of 40 studies, 4 studies (10\%) reported improvements in balance when the intervention was delivered via robotics [79], exergames [50], exergames in combination with health sensors [51], or video communication in combination with health sensors [52], when compared with physiotherapy [50,51,79] or usual care [52]. Another 2 studies (of 40,5\%) reported that either robotics [41] or exergames in combination with health sensors [46] could improve hand and arm function when compared with physiotherapy [41] or no intervention [46]. Pol and colleagues [48] found that patient-reported daily functioning significantly improved with the use of health sensors in combination with cognitive behavioral treatment, compared with cognitive behavioral treatment alone, reporting a difference of 1.17 (95\% CI $0.47-1.87 ; P<.001)$. Bernocchi and colleagues [52] reported that the use of video communication in combination with health sensors and a non-eHealth intervention was effective in preventing falls in patients who were at high risk of falling, when compared with usual care (29 falls vs 56 falls; $P<.001$ ). Of 40 studies, 1 study (3\%) demonstrated that the use of video communication in combination with health sensors improved physical activity when compared with usual care [53].

\section{Participation}

Of 40 studies, 12 studies (30\%) included 11 outcome measures within the participation domain $[40,43,45,48,50-53,57,64,67,79]$. Of these 12 studies, 3 studies (27\%) reported a significant difference in quality of life [52], mood [67], or self-assessment [48] when the intervention was delivered via the use of video communication in combination with health sensors and a non-eHealth intervention [52], virtual reality [67], or the use of health sensors in combination with a non-eHealth intervention [48]. Particularly, Bernocchi and colleagues [52] demonstrated that the use of video communication in combination with health sensors and a non-eHealth intervention significantly improved scores on the EuroQol Visual Analog Scale at 6 months, when compared with usual care (mean 63.8 vs mean $53.5 ; P<.001$ ). Koneva and colleagues [67] reported that the use of virtual reality decreased the severity of depression as measured by the Beck Depression Inventory, when compared with usual care (mean 9.5, SD 5.52 vs mean 10.3, SD 6.03; $P<.05$ ), Additionally, Pol and colleagues [48] found that the use of health sensors in combination with a non-eHealth intervention significantly improved the performance satisfaction in daily functioning at 6 months, when compared with usual care, reporting a difference of $0.94(95 \%$ CI $0.37-1.52 ; P<.001)$.

\section{External Factors}

Across all 40 studies, 5 studies (13\%) included outcome measures related to external factors $[40,42,45,61,63]$. Of these 5 studies, 2 studies $(40 \%)$ included robotics as interventions and found significant differences in cost, in favor of the intervention group [41,42]. Of the 5 studies, 1 study (20\%) included robotics as an intervention and found a difference in favor of the control group [45]. Hesse and colleagues [42] and Vanoglio and colleagues [41] reported decreases in cost with the use of robotics in comparison with either regular arm therapy (€4.15 [US \$4.92] for robotic interventions vs $€ 10.00$ [US $\$ 11.85$ ] for regular arm therapy, for each patient per session) [42] or physiotherapy ( $€ 237$ [US \$280.73] for robotic intervention vs $€ 480$ [US \$568.57] for physiotherapy, for each patient per 30 days) [41]. In contrast, Schoone and colleagues [45] reported an increase in total costs when compared with physiotherapy ( $€ 644.14$ [US $\$ 762.99$ ] for robotic interventions vs $€ 423.74$ [US \$501.93] for physiotherapy). Across all studies, no differences were found with regard to discharge settings [40,63], readmissions [40], or lengths of stay [61].

\section{Personal Factors}

Oesch and colleagues [49] found that self-regulated exercise using instruction leaflets was superior to exergames in terms of enjoyment (effect size: 0.88 , range $0.32-1.44 ; P<.001$ ) and motivation (effect size: 0.59 , range $0.05-1.14 ; P=.046$ ).

\section{Feasibility}

\section{Main Results for Feasibility}

Of the 40 included studies, 20 studies $(50 \%)$ evaluated the feasibility of the eHealth intervention used [41,46,50-52,54,57,59,60,62,64,65,69,71,72,74-78], of which 19 (of 20,95\%) concluded that the eHealth intervention was feasible when it was delivered via robotics [41,65,71,72], robotics in combination with exergames [74], exergames $[50,62,64,77]$, exergames in combination with health sensors [46,51], video communication [76], video communication in combination with health sensors [52,69], health sensors [57], health gateways in combination with health sensors [75], or mobile apps [54,59,60]. Peel and colleagues [78] reported that the use of video communication was not feasible due to problems related to patient limitations, staff issues, and the logistics of the system.

The outcome measures applied to evaluate feasibility varied considerably among studies, and a total of 19 different outcome measures were used. Of the 20 studies that reported feasibility, 6 studies (30\%) reported outcomes related to "adverse events," 7 studies (35\%) reported outcomes related to "adherence," and 7 studies (35\%) reported outcomes related to "exclusion rate." Another 4 studies (of 20, 20\%) did not specify the outcome measure used to evaluate feasibility but used outcomes related to effectiveness to establish feasibility $[54,64,65,72]$. 


\section{Adverse Events}

None of the included studies reported serious adverse events during the study period $[41,46,50,51,74,76]$. However, 2 studies (of 40,5\%) reported that some participants experienced discomfort during exergames [49,50].

\section{Adherence}

Of 40 studies, adherence was reported in 7 studies (18\%) [49-52,57,74], and 5 studies (13\%) reported information regarding the number of completed sessions [41,50-52,69]. Of the 7 studies reporting adherence, 5 studies (71\%) reported high levels of adherence, ranging from $76 \%$ [52] to $100 \%$ [74]. Of the 7 studies, 2 studies (29\%) reported low adherence in patients assigned to an exergame intervention when compared with either a non-eHealth intervention [49] or use of the exergame intervention below the recommended level $(<30$ minutes per week) [77].

\section{Exclusion Rate}

Of 40 studies, high exclusion rates were found in 7 studies (18\%). Specifically, of these 7 studies, 1 study (14\%) reported an exclusion rate of $64 \%$ [47], 2 studies (29\%) reported an exclusion rate of $75 \%$ [49,51], and 4 studies $(57 \%)$ reported an exclusion rate over $80 \%[42,45,50,68]$. In these latter studies, eHealth was delivered through complex eHealth interventions: robotics [42,45], exergames [50], and virtual reality [68]. The most commonly reported reasons for exclusion were cognitive impairment [45,47,49,51], physical impairment [45,49], and refusal to participate $[42,47,49-51,68]$. Of these 7 studies, in 2 studies (29\%), the reason given for declining to participate was "no interest" in eHealth [50,51].

\section{Usability}

\section{Main Results for Usability}

Of 40 studies, outcomes related to the usability of eHealth interventions were addressed in 4 studies (10\%): 2 studies (5\%) evaluated the usability of exergames [51,58], and another 2 studies $(5 \%)$ evaluated mobile apps [59,60]. Evaluation of usability consisted of a system usability scale [51], a survey of patients and therapists [58,59], or semistructured interviews $[59,60]$. Of the 4 studies that reported usability, 2 studies (50\%) included outcomes related to the barrier "cognition," 4 studies $(100 \%)$ included outcomes related to the aging barrier "motivation," and 1 study (25\%) included outcomes related to the barrier "physical ability." None of the studies included outcomes related to the barrier "perception."

\section{Cognition}

Ling and colleagues [58] reported that some patients found exergames too complicated because of the requirement to engage in multiple activities simultaneously, and they experienced difficulties in following instructions. To tailor the exergames to older patients with cognitive impairments, the authors advised to minimize the amount of information presented on the screen, which might help older patients to perceive the information better [58]. Additionally, White and colleagues [60] reported that patients with cognitive impairments experienced difficulties in operating mobile apps and needed their partner for support.

\section{Motivation}

Van den Berg and colleagues [51] reported a mean score of 62 (SD 21), on the system usability scale (scores ranging from 0 to 100), indicating that participants were generally comfortable with exergames and that they would like to use exergames more frequently. Similar findings were reported by Ling and colleagues [58], who concluded that patients and therapists both found exergames easy to use and therapists intended to use the exergame in the future. Therapists rated the exergame as highly satisfactory for motor rehabilitation in older patients after hip surgery. Findings regarding mobile apps indicated that patients readily grasped the skills required for use and that this was a beneficial source of extrinsic motivation $[59,60]$.

\section{Physical Ability}

Ling and colleagues [58] reported that some patients with physical disabilities had difficulties playing certain exergames that required stepping exercises because these patients were unable to maintain balance during exergames.

\section{Discussion}

\section{Principal Findings}

This review aimed to provide an overview of the effectiveness, feasibility, and usability of eHealth in geriatric rehabilitation. The review included 40 studies that applied eHealth interventions in older patients receiving geriatric rehabilitation. The majority of the included studies showed that eHealth interventions in geriatric rehabilitation are at least as effective as non-eHealth interventions. All studies that delivered eHealth in combination with another non-eHealth intervention reported positive outcomes. Most studies included outcome measures related to the ICF domain "activities." Very few studies included outcomes related to the ICF domain "participation." eHealth seems to be feasible in geriatric rehabilitation, since no serious adverse events were reported and most studies reported high levels of adherence. However, high exclusion rates were found in some studies. Results related to usability indicate that there are certain age-related barriers, such as cognition and physical ability, that lead to difficulties in using eHealth. Very few studies included outcomes related to feasibility and usability. However, these are important prerequisites to maximize the likelihood of successful implementation, and they thereby influence the effectiveness of eHealth.

\section{Comparison With Prior Work}

Our findings suggest that eHealth delivered via robotics, exergames, or health sensors is often found to be at least as effective as non-eHealth. Previous reviews that examined robotics [80], exergames [16], or health sensors [81,82] often found more beneficial results in favor of the intervention group. These reviews did not focus on older adults who were admitted for geriatric rehabilitation, and this could indicate that there are certain age-related barriers that affect the effectiveness of eHealth in older adults receiving geriatric rehabilitation. All of the included studies that delivered eHealth in combination with a non-eHealth intervention reported beneficial outcomes in favor of the intervention group. This is in line with other studies in which eHealth was delivered in combination with a non-eHealth 
intervention [83-85]. This indicates that eHealth is more beneficial when provided through blended care, where eHealth is delivered in combination with face-to-face treatment. This may provide a better quality of care by combining the best of the two types of interventions. This seems to especially be the case when blended care is delivered via video communication [52] or health sensors [48], since it offers the possibility to monitor and treat patients remotely.

Almost all of the studies that included outcomes related to feasibility concluded that eHealth was feasible in older adults receiving geriatric rehabilitation. None of the studies reported serious adverse events, which is in line with other reviews concerning feasibility of exergames $[15,86]$. The majority of the studies that included outcomes related to adherence or completed sessions reported high levels of adherence. Previous reviews that examined exergames also reported high adherence rates [86]. Some studies where eHealth was delivered via robotics or exergames reported a high exclusion rate (up to $88 \%$ ). All studies with exclusion rates of $\geq 75 \%$ were conducted in a geriatric rehabilitation setting $[45,49]$ or in a hospital with a dedicated rehabilitation unit [50,51]. Reasons for exclusion were mostly cognitive or physical impairments, problems that are often present in older patients receiving geriatric rehabilitation. These findings indicate that eHealth in geriatric rehabilitation is safe to use and overall adherence is expected to be high, but complex eHealth interventions such as robotics and exergames might only be feasible in a selective group of older patients receiving geriatric rehabilitation.

There is limited available evidence on the usability of eHealth interventions. The studies included in our review indicate that exergames and mobile apps are usable once older patients have been trained in their use. However, there were certain age-related barriers associated with cognitive or physical ability that led to difficulties in using eHealth. While we did not find studies that reported problems in the use of eHealth due to problems in perception, 2 of 4 studies ( $50 \%$ ) that included usability outcome measures explicitly excluded patients with visual impairments $[51,58]$. This might suggest that poor usability was expected in patients with visual impairments; this is in line with findings from other studies [27]. These findings suggest that usability problems are expected in older patients receiving geriatric rehabilitation, since they often suffer from cognitive, physical, or visual impairments. eHealth should be tailored to these specific age-related barriers to maximize the probability of successful use and implementation [22,27]. Furthermore, most studies did not incorporate clear usability endpoints, and the evaluation of usability varied considerably among studies. The lack of using clear endpoints or reliable and validated questionnaires combined with task metrics (preferably, task completion) to evaluate usability hampers the ability to pinpoint usability issues and prevents comparisons across different eHealth types $[25,87]$.

\section{Strengths and Limitations}

The first strength of this review is the extensive search strategy that covered a broad range of search databases and included all types of research designs. Another strength of this review is the categorization of outcome measures based on the ICF model, providing a clear overview of different types of outcome domains evaluated in the included studies. Nonetheless, several limitations of this systematic review should be noted. While this review provides a broad overview of the literature on 3 different concepts, our study design led to a vast variety of different outcome measures related to effectiveness. The inclusion of various outcomes measures, in combination with various eHealth interventions and diagnoses, limited our ability to draw definitive conclusions. Since a meta-analysis was not feasible, we were unable to report an effect size and publication bias. We instead provided an overview of the effectiveness of eHealth interventions using a harvest plot. Lastly, while we used a separate search string that included keywords related to usability, we only found 4 studies that included outcomes on usability. A possible explanation might be that we did not include specific Computer Science search databases, which might include more studies that are related to usability [88]. Furthermore, despite the massive growth in eHealth studies, only a small portion publish their usability results [89].

\section{Conclusions}

In conclusion, eHealth can improve rehabilitation outcomes in older adults receiving geriatric rehabilitation. Based on our findings, comparisons to literature, and the strengths and limitations of our review, our main results and recommendations for further research and the use of eHealth in clinical practice are (1) keep it simple, (2) include evidence on usability, (3) focus on participation, and (4) ensure consensus. First, simple interventions have the most potential to improve rehabilitation outcomes in older adults receiving geriatric rehabilitation, especially, when they are provided as blended care. Additionally, simple eHealth interventions have a higher chance of feasibility in older patients receiving geriatric rehabilitation who often suffer from cognitive or physical impairments. Second, scarce evidence on the usability of eHealth might hamper the implementation of eHealth in older patients receiving geriatric rehabilitation and could negatively influence effectiveness and feasibility. Further research on this topic with clear endpoints is needed. Health care professionals need to be aware of the usability of eHealth interventions they are providing. Third, participation is a key concept in geriatric rehabilitation and plays an important role in enabling older patients to continue living as independently as possible. Future research on eHealth interventions should consider including outcome measures related to participation. Fourth, current evidence on the use and evaluation of eHealth in geriatric rehabilitation is diverse, making it hard to compare outcomes and draw evident conclusions. Consensus on the use and evaluation of eHealth is needed for further development and implementation of eHealth in geriatric rehabilitation. 


\section{Authors' Contributions}

JJMK screened titles of the identified studies. JJMK and AV screened the abstracts of all potentially relevant studies and obtained and reviewed the full texts. Disagreements between JJMK and AV were discussed until a consensus was reached. If a disagreement could not be resolved, EFvDvI was consulted. JJMK extracted the data. AV also extracted a subset of the data (10\% of included studies) to check interrater reliability. JJMK performed quality assessment, and $10 \%$ of the included studies were selected at random and additionally assessed by AV to check interrater reliability. In 12 cases, a third reviewer, EFvDvI, was needed to achieve consensus during the process of study selection.

\section{Conflicts of Interest}

None declared.

\section{Multimedia Appendix 1}

PRISMA checklist.

[DOC File , 65 KB-Multimedia Appendix 1]

\section{Multimedia Appendix 2}

Keyword strings used for searching databases.

[PDF File (Adobe PDF File), 439 KB-Multimedia Appendix 2]

\section{Multimedia Appendix 3}

Detailed description of all included studies.

[XLSX File (Microsoft Excel File), 2794 KB-Multimedia Appendix 3]

\section{References}

1. United Nations. Department of Economic and Social affairs, Population division. World Population Ageing. 2019. URL: https://www.un.org/en/development/desa/population/publications/pdf/ageing/WorldPopulationAgeing2019-Report.pdf [accessed 2020-11-06]

2. Salive ME. Multimorbidity in older adults. Epidemiol Rev 2013 Jan 31;35(1):75-83. [doi: 10.1093/epirev/mxs009] [Medline: 23372025]

3. Inouye S, Studenski S, Tinetti M, Kuchel G. Geriatric syndromes: clinical, research, and policy implications of a core geriatric concept. J Am Geriatr Soc 2007 May;55(5):780-791 [FREE Full text] [doi: 10.1111/j.1532-5415.2007.01156.x] [Medline: 17493201$]$

4. Covinsky KE, Pierluissi E, Johnston CB. Hospitalization-associated disability: "She was probably able to ambulate, but I'm not sure". JAMA 2011 Oct 26;306(16):1782-1793. [doi: 10.1001/jama.2011.1556] [Medline: 22028354]

5. Bachmann S, Finger C, Huss A, Egger M, Stuck A, Clough-Gorr K. Inpatient rehabilitation specifically designed for geriatric patients: systematic review and meta-analysis of randomised controlled trials. BMJ 2010 Apr 20;340:c1718 [FREE Full text] [doi: 10.1136/bmj.c1718] [Medline: 20406866]

6. Holstege MS, Caljouw MA, Zekveld IG, van Balen R, de Groot AJ, van Haastregt JC, et al. Successful Geriatric Rehabilitation: Effects on Patients' Outcome of a National Program to Improve Quality of Care, the SINGER Study. J Am Med Dir Assoc 2017 May 01;18(5):383-387. [doi: 10.1016/j.jamda.2016.10.011] [Medline: 27939318]

7. Lettow B, Wouters M, Sinnige J. E-Health, wat is dat? Nictiz. URL: https://www.nictiz.nl/wp-content/uploads/ E-health-Wat-is-dat.pdf [accessed 2020-10-07]

8. Glasgow RE, Phillips SM, Sanchez MA. Implementation science approaches for integrating eHealth research into practice and policy. Int J Med Inform 2014 Jul;83(7):e1-11. [doi: 10.1016/j.ijmedinf.2013.07.002] [Medline: 23910896]

9. Ross J, Stevenson F, Lau R, Murray E. Factors that influence the implementation of e-health: a systematic review of systematic reviews (an update). Implement Sci 2016 Oct 26;11(1):146. [doi: 10.1186/s13012-016-0510-7] [Medline: 27782832]

10. Ossebaard HC, De Bruijn ACP, van Gemert-Pijnen JEWC, Geertsma RE. Risks related to the use of eHealth technologies: An exploratory study. National Institute for Public Health and the Environment. URL: https://www.rivm.nl/bibliotheek/ rapporten/360127001.pdf [accessed 2020-10-06]

11. Bonten TN, Rauwerdink A, Wyatt JC, Kasteleyn MJ, Witkamp L, Riper H, EHealth Evaluation Research Group. Online Guide for Electronic Health Evaluation Approaches: Systematic Scoping Review and Concept Mapping Study. J Med Internet Res 2020 Aug 12;22(8):e17774 [FREE Full text] [doi: 10.2196/17774] [Medline: 32784173]

12. Enam A, Torres-Bonilla J, Eriksson H. Evidence-Based Evaluation of eHealth Interventions: Systematic Literature Review. J Med Internet Res 2018 Nov 23;20(11):e10971 [FREE Full text] [doi: 10.2196/10971] [Medline: $\underline{30470678]}$ 
13. Broens THF, Huis in't Veld RMHA, Vollenbroek-Hutten MMR, Hermens HJ, van Halteren AT, Nieuwenhuis LJM. Determinants of successful telemedicine implementations: a literature study. J Telemed Telecare 2007 Jun;13(6):303-309. [doi: 10.1258/135763307781644951] [Medline: 17785027]

14. Muellmann S, Forberger S, Möllers T, Bröring E, Zeeb H, Pischke CR. Effectiveness of eHealth interventions for the promotion of physical activity in older adults: A systematic review. Prev Med 2018 Mar;108:93-110. [doi: 10.1016/j.ypmed.2017.12.026] [Medline: 29289643]

15. Skjæret N, Nawaz A, Morat T, Schoene D, Helbostad JL, Vereijken B. Exercise and rehabilitation delivered through exergames in older adults: An integrative review of technologies, safety and efficacy. Int J Med Inform 2016 Jan;85(1):1-16. [doi: 10.1016/j.ijmedinf.2015.10.008] [Medline: 26559887]

16. Zeng N, Pope Z, Lee J, Gao Z. A systematic review of active video games on rehabilitative outcomes among older patients. Journal of Sport and Health Science 2017 Mar;6(1):33-43 [FREE Full text] [doi: 10.1016/j.jshs.2016.12.002]

17. Reis E, Postolache G, Teixeira L, Arriaga P, Lima ML, Postolache O. Exergames for motor rehabilitation in older adults: an umbrella review. Physical Therapy Reviews 2019 Jul 19;24(3-4):84-99. [doi: 10.1080/10833196.2019.1639012]

18. Orsmond GI, Cohn ES. The Distinctive Features of a Feasibility Study: Objectives and Guiding Questions. OTJR (Thorofare N J) 2015 Jul 06;35(3):169-177. [doi: 10.1177/1539449215578649] [Medline: 26594739]

19. Matthew-Maich N, Harris L, Ploeg J, Markle-Reid M, Valaitis R, Ibrahim S, et al. Designing, Implementing, and Evaluating Mobile Health Technologies for Managing Chronic Conditions in Older Adults: A Scoping Review. JMIR mHealth uHealth 2016 Jun 09;4(2):e29. [doi: 10.2196/mhealth.5127] [Medline: 27282195]

20. Bowen DJ, Kreuter M, Spring B, Cofta-Woerpel L, Linnan L, Weiner D, et al. How we design feasibility studies. Am J Prev Med 2009 May;36(5):452-457 [FREE Full text] [doi: 10.1016/j.amepre.2009.02.002] [Medline: 19362699]

21. Kingston J. Conducting feasibility studies for knowledge based systems. Knowledge-Based Systems 2004 May;17(2-4):157-164. [doi: 10.1016/j.knosys.2004.03.011]

22. Hawley-Hague H, Boulton E, Hall A, Pfeiffer K, Todd C. Older adults' perceptions of technologies aimed at falls prevention, detection or monitoring: a systematic review. Int J Med Inform 2014 Jun;83(6):416-426 [FREE Full text] [doi: 10.1016/j.ijmedinf.2014.03.002] [Medline: 24798946]

23. Bevan N, Carter J, Earthy J, Geis T, Harker S. New ISO standards for usability, usability reports and usability measures. 2016 Jul 17 Presented at: Paper presented at: International conference on human-computer interaction; 2016; Toronto, Canada. [doi: 10.1007/978-3-319-39510-4_25]

24. Broekhuis M, Velsen L, Stal S, Weldink J, Tabak M. Why My Grandfather Finds Difficulty in using Ehealth: Differences in Usability Evaluations between Older Age Groups. In: Paper presented at: ICT4AW. 2019 Apr 23 Presented at: ICT4AW; 2019; prague czech republic p. E2019. [doi: 10.5220/0007680800480057]

25. Sousa V, Lopez KD. Towards Usable E-Health. Appl Clin Inform 2017 Dec 21;08(02):470-490. [doi: 10.4338/aci-2016-10-r-0170]

26. Alley SJ, Samra P, Rebar AL, Schoeppe S, Parkinson L, Power D, et al. A focus group study of older adults' perceptions and preferences towards web-based physical activity interventions. Inform Health Soc Care 2020 Sep 06;45(3):273-281. [doi: 10.1080/17538157.2019.1656210] [Medline: 31690152]

27. Wildenbos GA, Peute L, Jaspers M. Aging barriers influencing mobile health usability for older adults: A literature based framework (MOLD-US). Int J Med Inform 2018 Jun;114:66-75. [doi: 10.1016/j.ijmedinf.2018.03.012] [Medline: 29673606]

28. Demiris G, Finkelstein SM, Speedie SM. Considerations for the design of a Web-based clinical monitoring and educational system for elderly patients. J Am Med Inform Assoc 2001 Sep 01;8(5):468-472 [FREE Full text] [doi:

10.1136/jamia.2001.0080468] [Medline: 11522767]

29. Kraaijkamp J, van Dam van Isselt E, Chavannes N, Achterberg WP, Versluis A, Persoon A. Effectiveness of eHealth interventions in older patients who are submitted for geriatric rehabilitation: a protocol for a systematic review. PROSPERO International prospective register of systematic reviews. 2019. URL: https://www.crd.york.ac.uk/PROSPERO/display record. php?ID=CRD42019133192\&ID=CRD42019133192 [accessed 2019-12-01]

30. Moher D, Liberati A, Tetzlaff J, Altman D, PRISMA Group. Preferred reporting items for systematic reviews and meta-analyses: the PRISMA statement. Ann Intern Med 2009 Aug 18;151(4):264-9, W64 [FREE Full text] [doi: 10.7326/0003-4819-151-4-200908180-00135] [Medline: 19622511]

31. van Balen R, Gordon AL, Schols JMGA, Drewes YM, Achterberg WP. What is geriatric rehabilitation and how should it be organized? A Delphi study aimed at reaching European consensus. Eur Geriatr Med 2019 Oct 08;10(6):977-987. [doi: 10.1007/s41999-019-00244-7]

32. Grund S, Gordon AL, van Balen R, Bachmann S, Cherubini A, Landi F, et al. European consensus on core principles and future priorities for geriatric rehabilitation: consensus statement. Eur Geriatr Med 2020 Apr 13;11(2):233-238. [doi: 10.1007/s41999-019-00274-1] [Medline: 32297191]

33. WHO. International classification of functioning, disability and health. WHO. International classification of functioning, disabilityhealth: World Health Organization; 2001. URL: http://apps.who.int/iris/bitstream/handle/10665/42407/9241545429. pdf:jsessionid=DD34E52C5D093BFA4F006375315882CE?sequence=1 [accessed 2020-12-19]

34. Vertias Health Innovation. Covidence systematic review software. In: Veritas Health Innovation Melbourne, VIC; 2019. URL: https://www.covidence.org/ [accessed 2019-10-10] 
35. Krijgsman J, Klein WG. Ordening in de wereld van eHealth. Nictiz. 2012. URL: https://www.nictiz.nl/wp-content/uploads/ 2012/08/Whitepaper-Ordening-in-de-wereld-van-eHealth.pdf [accessed 2019-10-11]

36. Hong QN, Fàbregues S, Bartlett G, Boardman F, Cargo M, Dagenais P, et al. The Mixed Methods Appraisal Tool (MMAT) version 2018 for information professionals and researchers. EFI 2018 Dec 18;34(4):285-291. [doi: 10.3233/EFI-180221]

37. Higgins J, Green S. Cochrane handbook for systematic reviews of interventions. Vol 4: John Wiley \& Sons; Feb 1, 2011.

38. Thomas B, Ciliska D, Dobbins M, Micucci S. A Process for Systematically Reviewing the Literature: Providing the Research Evidence for Public Health Nursing Interventions. Worldviews on Evidence-Based Nursing 2004 Jun 23;1(3):176-184. [doi: $10.1111 / \mathrm{j} .1524-475 x .2004 .04006 . x]$

39. Ogilvie D, Fayter D, Petticrew M, Sowden A, Thomas S, Whitehead M, et al. The harvest plot: a method for synthesising evidence about the differential effects of interventions. BMC Med Res Methodol 2008 Feb 25;8(1):8 [FREE Full text] [doi: 10.1186/1471-2288-8-8] [Medline: 18298827$]$

40. Peel NM, Paul SK, Cameron ID, Crotty M, Kurrle SE, Gray LC. Promoting Activity in Geriatric Rehabilitation: A Randomized Controlled Trial of Accelerometry. PLoS One 2016 Aug 26;11(8):e0160906 [FREE Full text] [doi: 10.1371/journal.pone.0160906] [Medline: 27564857]

41. Vanoglio F, Bernocchi P, Mulè C, Garofali F, Mora C, Taveggia G, et al. Feasibility and efficacy of a robotic device for hand rehabilitation in hemiplegic stroke patients: a randomized pilot controlled study. Clin Rehabil 2017 Mar 10;31(3):351-360. [doi: 10.1177/0269215516642606] [Medline: 27056250]

42. Hesse S, Heß A, Werner C, Kabbert N, Buschfort R. Effect on arm function and cost of robot-assisted group therapy in subacute patients with stroke and a moderately to severely affected arm: a randomized controlled trial. Clin Rehabil 2014 Jul 22;28(7):637-647. [doi: 10.1177/0269215513516967] [Medline: 24452706]

43. Taveggia G, Borboni A, Mulé C, Villafañe JH, Negrini S. Conflicting results of robot-assisted versus usual gait training during postacute rehabilitation of stroke patients: a randomized clinical trial. Int J Rehabil Res 2016 Mar;39(1):29-35 [FREE Full text] [doi: 10.1097/MRR.0000000000000137] [Medline: 26512928]

44. Cannell J, Jovic E, Rathjen A, Lane K, Tyson AM, Callisaya ML, et al. The efficacy of interactive, motion capture-based rehabilitation on functional outcomes in an inpatient stroke population: a randomized controlled trial. Clin Rehabil 2018 Feb 19;32(2):191-200 [FREE Full text] [doi: 10.1177/0269215517720790] [Medline: 28719977]

45. Schoone M, Dusseldorp E, van den Akker-van Marle M, Doornebosch A, Bal R, Meems A, et al. Stroke Rehabilitation in Frail Elderly with the Robotic Training Device ACRE: A Randomized Controlled Trial and Cost-Effectiveness Study. Journal of Robotics 2011;2011:1-10. [doi: 10.1155/2011/543060]

46. Iosa M, Morone G, Fusco A, Castagnoli M, Fusco FR, Pratesi L, et al. Leap motion controlled videogame-based therapy for rehabilitation of elderly patients with subacute stroke: a feasibility pilot study. Topics in Stroke Rehabilitation 2015 Feb 25;22(4):306-316. [doi: 10.1179/1074935714z.0000000036]

47. Piqueras M, Marco E, Coll M, Escalada F, Ballester A, Cinca C, et al. Effectiveness of an interactive virtual telerehabilitation system in patients after total knee arthoplasty: a randomized controlled trial. J Rehabil Med 2013 Apr;45(4):392-396 [FREE Full text] [doi: 10.2340/16501977-1119] [Medline: 23474735]

48. Pol M, Ter Riet G, van Hartingsveldt M, Kröse B, Buurman B. Effectiveness of sensor monitoring in a rehabilitation programme for older patients after hip fracture: a three-arm stepped wedge randomised trial. Age Ageing 2019 Sep 01;48(5):650-657. [doi: 10.1093/ageing/afz074] [Medline: $\underline{\text { 31204776] }}$

49. Oesch P, Kool J, Fernandez-Luque L, Brox E, Evertsen G, Civit A, et al. Exergames versus self-regulated exercises with instruction leaflets to improve adherence during geriatric rehabilitation: a randomized controlled trial. BMC Geriatr 2017 Mar 23;17(1):77 [FREE Full text] [doi: 10.1186/s12877-017-0467-7] [Medline: 28330455]

50. Laver K, George S, Ratcliffe J, Quinn S, Whitehead C, Davies O, et al. Use of an interactive video gaming program compared with conventional physiotherapy for hospitalised older adults: a feasibility trial. Disabil Rehabil 2012 Mar 12;34(21):1802-1808. [doi: 10.3109/09638288.2012.662570] [Medline: 22409245]

51. van den Berg M, Sherrington C, Killington M, Smith S, Bongers B, Hassett L, et al. Video and computer-based interactive exercises are safe and improve task-specific balance in geriatric and neurological rehabilitation: a randomised trial. J Physiother 2016 Jan;62(1):20-28 [FREE Full text] [doi: 10.1016/j.jphys.2015.11.005] [Medline: 26701163]

52. Bernocchi P, Giordano A, Pintavalle G, Galli T, Ballini Spoglia E, Baratti D, et al. Feasibility and Clinical Efficacy of a Multidisciplinary Home-Telehealth Program to Prevent Falls in Older Adults: A Randomized Controlled Trial. J Am Med Dir Assoc 2019 Mar;20(3):340-346. [doi: 10.1016/j.jamda.2018.09.003] [Medline: 30366759]

53. Barnason S, Zimmerman L, Schulz P, Tu C. Influence of an early recovery telehealth intervention on physical activity and functioning after coronary artery bypass surgery among older adults with high disease burden. Heart Lung 2009 Nov;38(6):459-468 [FREE Full text] [doi: 10.1016/j.hrtlng.2009.01.010] [Medline: 19944870]

54. Li CT, Hung GK, Fong KN, Gonzalez PC, Wah S, Tsang HW. Effects of a home-based occupational therapy telerehabilitation via smartphone for outpatients after hip fracture surgery: A feasibility randomised controlled study. J Telemed Telecare 2020 Jun 28:1357633X2093243. [doi: 10.1177/1357633x20932434]

55. Karner S, Stenner H, Spate M, Behrens J, Krakow K. Effects of a robot intervention on visuospatial hemineglect in postacute stroke patients: a randomized controlled trial. Clin Rehabil 2019 Dec 13;33(12):1940-1948. [doi: 10.1177/0269215519865993] [Medline: $\underline{\text { 31409126] }}$ 
56. Franceschini M, Mazzoleni S, Goffredo M, Pournajaf S, Galafate D, Criscuolo S, et al. Upper limb robot-assisted rehabilitation versus physical therapy on subacute stroke patients: A follow-up study. J Bodyw Mov Ther 2020 Jan;24(1):194-198. [doi: 10.1016/j.jbmt.2019.03.016] [Medline: 31987544]

57. Da-Silva RH, Moore SA, Rodgers H, Shaw L, Sutcliffe L, van Wijck F, et al. Wristband Accelerometers to motiVate arm Exercises after Stroke (WAVES): a pilot randomized controlled trial. Clin Rehabil 2019 Aug 07;33(8):1391-1403. [doi: 10.1177/0269215519834720] [Medline: 30845829]

58. Ling Y, Ter Meer LP, Yumak Z, Veltkamp RC. Usability Test of Exercise Games Designed for Rehabilitation of Elderly Patients After Hip Replacement Surgery: Pilot Study. JMIR Serious Games 2017 Oct 12;5(4):e19 [FREE Full text] [doi: 10.2196/games.7969] [Medline: 29025696]

59. Backman C, Harley A, Kuziemsky C, Mercer J, Peyton L. MyPath to Home Web-Based Application for the Geriatric Rehabilitation Program at Bruyère Continuing Care: User-Centered Design and Feasibility Testing Study. JMIR Form Res 2020 Sep 14;4(9):e18169 [FREE Full text] [doi: 10.2196/18169] [Medline: 32924953]

60. White J, Janssen H, Jordan L, Pollack M. Tablet technology during stroke recovery: a survivor's perspective. Disabil Rehabil 2015 Sep 12;37(13):1186-1192. [doi: 10.3109/09638288.2014.958620] [Medline: 25212736]

61. Dakin L, Peel N. Effect of accelerometry on the functional mobility of older rehabilitation inpatients as measured by functional independence measure--locomotion (FIM) gain: a retrospective matched cohort study. J Nutr Health Aging 2011 May 30;15(5):382-386. [doi: 10.1007/s12603-010-0129-3] [Medline: 21528165]

62. Chan T, Chan F, Shea Y, Lin O, Luk J, Chan F. Interactive virtual reality Wii in geriatric day hospital: a study to assess its feasibility, acceptability and efficacy. Geriatr Gerontol Int 2012 Oct;12(4):714-721. [doi: 10.1111/j.1447-0594.2012.00848.x] [Medline: 22469232]

63. Hicks SA, Cimarolli VR. The effects of telehealth use for post-acute rehabilitation patient outcomes. J Telemed Telecare 2016 Dec 26;24(3):179-184. [doi: 10.1177/1357633x16686771]

64. Levinger P, Zeina D, Teshome AK, Skinner E, Begg R, Abbott JH. A real time biofeedback using Kinect and Wii to improve gait for post-total knee replacement rehabilitation: a case study report. Disabil Rehabil Assist Technol 2016 Sep 04;11(3):251-262. [doi: 10.3109/17483107.2015.1080767] [Medline: 26336875]

65. Goto K, Morishita T, Kamada S, Saita K, Fukuda H, Shiota E, et al. Feasibility of rehabilitation using the single-joint hybrid assistive limb to facilitate early recovery following total knee arthroplasty: A pilot study. Assist Technol 2017 Sep 30;29(4):197-201. [doi: 10.1080/10400435.2016.1219883] [Medline: 27689789]

66. Yoshikawa K, Mutsuzaki H, Sano A, Koseki K, Fukaya T, Mizukami M, et al. Training with Hybrid Assistive Limb for walking function after total knee arthroplasty. J Orthop Surg Res 2018 Jul 03;13(1):163 [FREE Full text] [doi: 10.1186/s13018-018-0875-1] [Medline: 29970139]

67. Koneva E, Timashkova G, Shapovalenko T, Lyadov K. Functional Spatially-Oriented Rehabilitation Of Elderly Patients After Cerebral Stroke. Reseasrch Journal of Pharmaceutical, Biological, and Chemical Sciences 2018;9(5):2232-2238.

68. Edmans J, Gladman J, Hilton D, Walker M, Sunderland A, Cobb S, et al. Clinical evaluation of a non-immersive virtual environment in stroke rehabilitation. Clin Rehabil 2009 Feb 01;23(2):106-116. [doi: 10.1177/0269215508095875] [Medline: $\underline{19164398]}$

69. Bernocchi P, Vanoglio F, Baratti D, Morini R, Rocchi S, Luisa A, et al. Home-based telesurveillance and rehabilitation after stroke: a real-life study. Top Stroke Rehabil 2016 Apr 09;23(2):106-115. [doi: 10.1080/10749357.2015.1120453] [Medline: 27078116 ]

70. Doornebosch AJ, Cools HJ, Slee-Turkenburg ME, van Elk MG, Schoone-Harmsen M. Robot-mediated ACtive REhabilitation (ACRE2) for the hemiplegic upper limb after a stroke: A pilot study. TAD 2008 Jan 03;19(4):199-203. [doi: 10.3233/tad-2007-19405]

71. Gandolfi M, Geroin C, Tomelleri C, Maddalena I, Kirilova Dimitrova E, Picelli A, et al. Feasibility and safety of early lower limb robot-assisted training in sub-acute stroke patients: a pilot study. Eur J Phys Rehabil Med 2017 Dec;53(6):870-882. [doi: 10.23736/s1973-9087.17.04468-9]

72. Hesse $\mathrm{S}$, Waldner A, Tomelleri C. Innovative gait robot for the repetitive practice of floor walking and stair climbing up and down in stroke patients. J Neuroeng Rehabil 2010 Jun 28;7(1):30 [FREE Full text] [doi: 10.1186/1743-0003-7-30] [Medline: 20584307]

73. Sampson M, Shau Y, King MJ. Bilateral upper limb trainer with virtual reality for post-stroke rehabilitation: case series report. Disabil Rehabil Assist Technol 2012 Mar 29;7(1):55-62. [doi: 10.3109/17483107.2011.562959] [Medline: 21446826]

74. Schwickert L, Klenk J, Stähler A, Becker C, Lindemann U. Robotic-assisted rehabilitation of proximal humerus fractures in virtual environments: a pilot study. Z Gerontol Geriatr 2011 Dec 14;44(6):387-392. [doi: 10.1007/s00391-011-0258-2] [Medline: 22159833]

75. Marschollek M, Becker M, Bauer JM, Bente P, Dasenbrock L, Elbers K, et al. Multimodal activity monitoring for home rehabilitation of geriatric fracture patients--feasibility and acceptance of sensor systems in the GAL-NATARS study. Inform Health Soc Care 2014 Aug 22;39(3-4):262-271. [doi: 10.3109/17538157.2014.931852] [Medline: 25148561]

76. Tousignant M, Boissy P, Corriveau H, Moffet H. In home telerehabilitation for older adults after discharge from an acute hospital or rehabilitation unit: A proof-of-concept study and costs estimation. Disabil Rehabil Assist Technol 2006 Sep 09;1(4):209-216. [doi: 10.1080/17483100600776965] [Medline: 19260168] 
77. Cimarolli VR, Reinhardt JP, Minahan J, Burack O, Thomas C, Melly R. Use of an Exercise Technology in Post-Acute Care of a Skilled Nursing Facility: A Feasibility Study. J Am Med Dir Assoc 2017 Nov 01;18(11):991.e1-991.e4. [doi: 10.1016/j.jamda.2017.07.002] [Medline: 28843523]

78. Peel NM, Russell TG, Gray LC. Feasibility of using an in-home video conferencing system in geriatric rehabilitation. $\mathrm{J}$ Rehabil Med 2011 Mar;43(4):364-366 [FREE Full text] [doi: 10.2340/16501977-0675] [Medline: 21305228]

79. Takano E, Ozaki K, Satoh K, Kawamura K, Maltais M, Kondo I. Effects of a Balance Exercise Assist Robot on Older Patients with Hip Fracture: A Preliminary Study. J. Med. Biol. Eng 2020 Oct 08;40(6):783-789. [doi: 10.1007/s40846-020-00568-x]

80. Mehrholz J, Pohl M, Platz T, Kugler J, Elsner B. Electromechanical and robot-assisted arm training for improving activities of daily living, arm function, and arm muscle strength after stroke. Cochrane Database Syst Rev 2018 Sep 03;9:CD006876. [doi: 10.1002/14651858.CD006876.pub5] [Medline: $\underline{\text { 30175845] }}$

81. Cooper C, Gross A, Brinkman C, Pope R, Allen K, Hastings S, et al. The impact of wearable motion sensing technology on physical activity in older adults. Exp Gerontol 2018 Oct 02;112:9-19 [FREE Full text] [doi: 10.1016/j.exger.2018.08.002] [Medline: $\underline{30103025]}$

82. Goode AP, Hall KS, Batch BC, Huffman KM, Hastings SN, Allen KD, et al. The Impact of Interventions that Integrate Accelerometers on Physical Activity and Weight Loss: A Systematic Review. Ann Behav Med 2017 Feb 26;51(1):79-93 [FREE Full text] [doi: 10.1007/s12160-016-9829-1] [Medline: 27565168]

83. Talboom-Kamp EPWA, Verdijk NA, Kasteleyn MJ, Harmans LM, Talboom IJSH, Numans ME, et al. Effect of a combined education and eHealth programme on the control of oral anticoagulation patients (PORTALS study): a parallel cohort design in Dutch primary care. BMJ Open 2017 Sep 27;7(9):e017909 [FREE Full text] [doi: 10.1136/bmjopen-2017-017909] [Medline: 28963313]

84. Wentzel J, van der Vaart R, Bohlmeijer ET, van Gemert-Pijnen JEWC. Mixing Online and Face-to-Face Therapy: How to Benefit From Blended Care in Mental Health Care. JMIR Ment Health 2016 Feb 09;3(1):e9 [FREE Full text] [doi: 10.2196/mental.4534] [Medline: 26860537]

85. van der Kleij RM, Kasteleyn MJ, Meijer E, Bonten TN, Houwink EJ, Teichert M, et al. SERIES: eHealth in primary care. Part 1: Concepts, conditions and challenges. Eur J Gen Pract 2019 Oct 10;25(4):179-189 [FREE Full text] [doi: 10.1080/13814788.2019.1658190] [Medline: 31597502]

86. Valenzuela T, Okubo Y, Woodbury A, Lord SR, Delbaere K. Adherence to Technology-Based Exercise Programs in Older Adults: A Systematic Review. J Geriatr Phys Ther 2018 Jun 29;41(1):49-61. [doi: 10.1519/JPT.0000000000000095] [Medline: $\underline{27362526}$ ]

87. Broekhuis M, van Velsen L, Hermens H. Assessing usability of eHealth technology: A comparison of usability benchmarking instruments. Int J Med Inform 2019 Aug;128:24-31 [FREE Full text] [doi: 10.1016/j.ijmedinf.2019.05.001] [Medline: 31160008]

88. Zapata BC, Fernández-Alemán JL, Idri A, Toval A. Empirical studies on usability of mHealth apps: a systematic literature review. J Med Syst 2015 Feb;39(2):1. [doi: 10.1007/s10916-014-0182-2] [Medline: 25600193]

89. Maramba I, Chatterjee A, Newman C. Methods of usability testing in the development of eHealth applications: A scoping review. Int J Med Inform 2019 Jun;126:95-104 [FREE Full text] [doi: 10.1016/j.ijmedinf.2019.03.018] [Medline: 31029270]

\section{Abbreviations}

ICF: International Classification of Functioning, Disability, and Health.

MMAT: Mixed Methods Appraisal Tool.

PRISMA: Preferred Reporting Items for Systematic Reviews and Meta-analyses.

Edited by G Eysenbach; submitted 31.08.20; peer-reviewed by N Skjaeret, M Broekhuis, S Bhattacharjya; comments to author 19.12.20;
revised version received 11.02.21; accepted 16.05.21; published 19.08.21
Please cite as:
Kraaijkamp JJM, van Dam van Isselt EF, Persoon A, Versluis A, Chavannes NH, Achterberg WP
eHealth in Geriatric Rehabilitation: Systematic Review of Effectiveness, Feasibility, and Usability
J Med Internet Res 2021;23(8):e24015
URL: $\underline{\text { https://www.jmir.org/2021/8/e24015 }}$
doi: $\underline{10.2196 / 24015}$
PMID: $\underline{34420918}$

CJules J M Kraaijkamp, Eléonore F van Dam van Isselt, Anke Persoon, Anke Versluis, Niels H Chavannes, Wilco P Achterberg. Originally published in the Journal of Medical Internet Research (https://www.jmir.org), 19.08.2021. This is an open-access 
article distributed under the terms of the Creative Commons Attribution License (https://creativecommons.org/licenses/by/4.0/), which permits unrestricted use, distribution, and reproduction in any medium, provided the original work, first published in the Journal of Medical Internet Research, is properly cited. The complete bibliographic information, a link to the original publication on https://www.jmir.org/, as well as this copyright and license information must be included. 